\title{
Modeling the excitation of graphene plasmons in periodic grids of graphene ribbons: An analytical approach
}

\author{
Gonçalves, P:A.D.; Dias, E. J. C.; Bludov, Yu V.; Peres, N. M. R.
}

Published in:

Physical Review B

Link to article, DOI:

10.1103/PhysRevB.94.195421

Publication date:

2016

Document Version

Publisher's PDF, also known as Version of record

Link back to DTU Orbit

Citation $(A P A)$ :

Gonçalves, PA. D., Dias, E. J. C., Bludov, Y. V., \& Peres, N. M. R. (2016). Modeling the excitation of graphene plasmons in periodic grids of graphene ribbons: An analytical approach. Physical Review B, 94(19), [195421]. https://doi.org/10.1103/PhysRevB.94.195421

\section{General rights}

Copyright and moral rights for the publications made accessible in the public portal are retained by the authors and/or other copyright owners and it is a condition of accessing publications that users recognise and abide by the legal requirements associated with these rights.

- Users may download and print one copy of any publication from the public portal for the purpose of private study or research.

- You may not further distribute the material or use it for any profit-making activity or commercial gain

- You may freely distribute the URL identifying the publication in the public portal 


\title{
Modeling the excitation of graphene plasmons in periodic grids of graphene ribbons: An analytical approach
}

\author{
P. A. D. Gonçalves ${ }^{1,2,3,{ }^{*}}$ E. J. C. Dias, ${ }^{1}$ Yu. V. Bludov,${ }^{1}$ and N. M. R. Peres ${ }^{1, \dagger}$ \\ ${ }^{1}$ Department of Physics and Center of Physics, University of Minho, PT-4710-057, Braga, Portugal \\ ${ }^{2}$ Department of Photonics Engineering, Technical University of Denmark, DK-2800 Kongens Lyngby, Denmark \\ ${ }^{3}$ Center for Nanostructured Graphene (CNG), Technical University of Denmark, DK-2800 Kongens Lyngby, Denmark
}

(Received 25 July 2016; published 14 November 2016)

\begin{abstract}
We study electromagnetic scattering and subsequent plasmonic excitations in periodic grids of graphene ribbons. To address this problem, we develop an analytical method to describe the plasmon-assisted absorption of electromagnetic radiation by a periodic structure of graphene ribbons forming a diffraction grating for $\mathrm{THz}$ and mid-IR light. The major advantage of this method lies in its ability to accurately describe the excitation of graphene surface plasmons (GSPs) in one-dimensional (1D) graphene gratings without the use of both time-consuming, and computationally demanding full-wave numerical simulations. We thus provide analytical expressions for the reflectance, transmittance, and plasmon-enhanced absorbance spectra, which can be readily evaluated using any personal computer with little to no programming. We also introduce a semianalytical method to benchmark our previous results and further compare the theoretical data with spectra taken from experiments, for which we observe a very good agreement. These theoretical tools may therefore be applied to design new experiments and cutting-edge nanophotonic devices based on graphene plasmonics.
\end{abstract}

DOI: 10.1103/PhysRevB.94.195421

\section{INTRODUCTION}

Nowadays, photonics—dubbed "the science of light"-is one of the branches of the physical sciences with the most impact in our daily lives. It is concerned with the study and manipulation of light (photons) in a manifold of fundamental and technological landscapes. Recently, the "nanorevolution" under way has led to the miniaturization of electronics. However, regarding electromagnetic (EM) radiation, such miniaturization is limited by the length scale defined by the wavelength of the employed light (known as the diffraction limit). In this context, plasmonics [1] has been regarded as the most promising candidate to bring EM fields to the nanoscale [2-6]. Plasmonics is a branch of photonics which deals with quasiparticles known as plasmon polaritons [1,7]. Surface plasmon polaritons (SPPs) are electromagnetic surface waves coupled to collective excitations of the free electrons in conductors. When these hybrid excitations occur in conducting nanostructures-such as nanoparticles $[8,9]$ or engineered metamaterials $[10,11]$ the corresponding nonpropagating plasmon polaritons are generally referred to as localized surface plasmons (LSPs) [1]. Perhaps the most alluring property of plasmons is that they exhibit large field enhancements and deep subwavelength confinement of EM fields, thereby circumventing the diffraction limit of conventional optics [2-5]. For this reason, plasmonics has been considered the ultimate pathway to manipulate light-matter interactions at the nanometer scale.

Very recently, graphene [12-14] - a two-dimensional (2D) crystal made up of carbon atoms arranged in a honeycomb lattice-has emerged as a promising plasmonic material, benefiting from this material's remarkable electronic and optical properties [13-16]. Doped graphene is capable of supporting SPPs - graphene surface plasmon polaritons (GSPs)

\footnotetext{
*padgo@fotonik.dtu.dk

†peres@ fisica.uminho.pt
}

[17-24]—in the THz and mid-IR spectral range. These possess tantalizing properties, outperforming traditional noble-metal plasmonics, in that spectral window, in terms of mode confinement, and are predicted to suffer from relatively low losses when compared to conventional three-dimensional (3D) metals $[18,19,21,25]$. In addition, graphene plasmons have yet another key advantage: the ability to be actively tunable by means of electrical gating or chemical doping. This feature constitutes a major improvement over conventional metalbased plasmonics [1,7] (where tunability is usually limited by the geometry and composition of the system, and therefore it is fixed), and constitutes a sought-after characteristic for active nanophotonic devices and/or circuitry based on graphene plasmons. Indeed, a plethora of proof-of-concept, application-oriented experiments have already demonstrated the capabilities of GSPs to deliver extremely sensitive biochemical sensors [26-30], surface-enhanced Raman scattering (SERS) [31-36], polarizers [37,38], optical modulators [3942], and photodetectors [43-47].

Such achievements are particularly notable considering that optical excitation of graphene plasmons was only achieved as recently as in 2011 by $\mathrm{Ju}$ et al., using periodic arrays of graphene microribbons [48]. That foundational publication paved the way for the emergence of many experimental and theoretical works that soon followed, thereby establishing the field of graphene plasmonics [17-24]. As of today, GSPs have been realized in a number of systems, ranging from patterned grids of graphene ribbons [26,27,38,40,4853], disks [51,54-57], and rings [54,55], periodic antidot lattices [57-59], resonators [60,61], and hybrid graphenemetal nanoantennas [43,44,62-64], among others [65-74].

In the heart of plasmonics lies the fact that freely propagating EM radiation cannot couple directly to plasmons owing to the momentum mismatch between plasmons and photons of the same frequency. However, the property that the plasmon's wave vector is larger than the wave vector of light of the same frequency is exactly what enables extreme 
localization of light into subwavelength volumes. For extended graphene, these volumes can be about $\alpha^{3} \approx 10^{-6}$ times smaller (where $\alpha$ denotes the fine-structure constant) than the volume characterized by the free-space light's wavelength (i.e., $\lambda_{0}^{-3}$ ). Typical strategies to couple light to graphene plasmons involve the patterning of pristine graphene into gratings and related nanostructures [26,27,38,40,48-51,51-57,57-59], the use of dielectric gratings $[65,66]$, light scattering from a conductive tip [71-74], and even nonlinear three-wave mixing [69,70].

In this context, the utilization of periodic grids of graphene ribbons-fabricated by patterning an otherwise continuous graphene sheet-has been one of the most popular setups to realize graphene plasmons with energies from the $\mathrm{THz}$ up to the mid-IR regime, which can be tailored either by varying the size of the ribbons or by tuning the concentration of charge carriers in graphene (and thus the Fermi level). Under this scheme, the array of graphene ribbons effectively acts as a diffraction grating for EM radiation impinging on the system (e.g., from a laser), producing scattered waves which carry momenta in multiples of the reciprocal lattice vector, $G=$ $2 \pi / L$ (where $L$ is the grating period), thus overcoming the above-mentioned kinematic constraint. The reason the use of ribbon arrays to couple light to GSPs has been so predominant is essentially twofold (apart from being easily attainable with current fabrication technologies): it enables us to overcome the momentum mismatch between light and GSPs, and it renders a stronger (composite) plasmonic response than one would get from a single graphene ribbon (also, in this latter case, instead of well-defined diffracted orders, the scattered waves would transport a continuum of momenta).

In this work we develop an analytical framework describing the interaction of EM radiation with periodic grids of micro- and nanosized graphene ribbons. The main motivation driving this work was to deliver a simple and transparent theoretical tool capable of explaining the plasmon-induced spectra measured in experiments that did not involve the use of computationally heavy and time-consuming numerical simulations. Here, we provide simple closed-form expressions for the reflectance, transmittance, and absorbance spectra of THz and mid-IR light through graphene patterned into ribbons. These spectra may then be used to design or model experiments with graphene plasmons in the laboratory, by simply evaluating an analytical expression. The coupling between graphene plasmons and surface optical ( $\mathrm{SO}$ ) phonon modes of a $\mathrm{SiO}_{2}$ substrate is also considered, and we observe a reconstruction of the polaritonic spectrum owing to the hybridization of GSPs with SO phonons of the underlying polar substrate. We further introduce a semianalytical technique developed elsewhere [22,24,75-77] to benchmark our analytical theory. Finally, we compare the outcomes of both frameworks against actual experimental data and demonstrate their ability to describe plasmonic excitations in periodic gratings of graphene ribbons.

\section{THEORY}

\section{A. Analytical method}

We consider the scattering of EM radiation by a $1 \mathrm{D}$ periodic grid of graphene ribbons of width $w$. For the sake of simplicity,

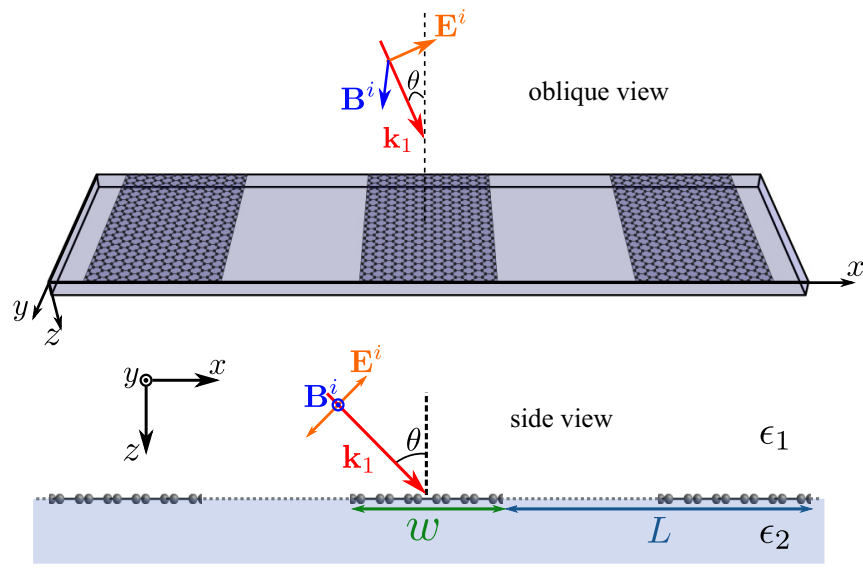

FIG. 1. Monochromatic p-polarized plane-wave impinging on a grid of graphene ribbons (not to scale) arranged in a grating-like configuration. The ribbons are sitting in the plane defined by $z=0$. The structure is periodic, with period $L$, and the width of the graphene ribbons is defined by $w$. The system is encapsulated between a top insulator with relative permittivity $\epsilon_{1}$ (for $z<0$ ) and a dielectric substrate with relative permittivity $\epsilon_{2}$ (for $z>0$ ).

the ribbons are assumed to possess infinite length in the longitudinal direction. In such an arrangement, the graphene grid behaves like a diffraction grating for EM waves. The period of the grating is denoted by $L$ hereafter, and the system is assumed to lie in the plane defined by $z=0$, being cladded between two dielectric media with relative permittivities $\epsilon_{1}$ (for $z<0$ ) and $\epsilon_{2}$ (for $z>0$ ); see Fig. 1. In what follows, we assume ribbons whose widths are $\sim 100 \mathrm{~nm}$ or larger, so that the actual edge termination of the graphene ribbons and finite-sized effects are not important, and therefore a classical electrodynamics framework suffices [78,79].

We consider a $p$-polarized monochromatic plane wave impinging on the grid of graphene ribbons at an angle $\theta$. For such polarization, the incident EM fields read

$$
\begin{gathered}
\mathbf{B}^{i}(\mathbf{r}, t)=B_{0}^{i} e^{i\left(\mathbf{k}_{1} \cdot \mathbf{r}-\omega t\right)} \hat{\mathbf{y}} \\
\mathbf{E}^{i}(\mathbf{r}, t)=\left(E_{0, x}^{i} \hat{\mathbf{x}}+E_{0, z}^{i} \hat{\mathbf{z}}\right) e^{i\left(\mathbf{k}_{1} \cdot \mathbf{r}-\omega t\right)},
\end{gathered}
$$

where the wave vector of the incoming wave is defined as $\mathbf{k}_{1}=$ $k_{x} \hat{\mathbf{x}}+k_{z} \hat{\mathbf{z}}$, with $k_{x}=\sqrt{\epsilon_{1}} k_{0} \sin \theta$ and $k_{z}=\sqrt{\epsilon_{1}} k_{0} \cos \theta$, where $k_{0}=\omega / c$. Naturally, the field amplitudes $B_{0}^{i}, E_{0, x}^{i}$, and $E_{0, z}^{i}$ are connected via Maxwell's equations, which establish the relations $E_{0, x}^{i}=\frac{c^{2} k_{z}}{\omega \epsilon_{1}} B_{0}^{i}$ and $E_{0, z}^{i}=-\frac{c^{2} k_{x}}{\omega \epsilon_{1}} B_{0}^{i}$. Furthermore, due the periodicity of the grid, one may write the reflected magnetic field in the form of a Bloch sum (also termed as Fourier-Floquet decomposition),

$$
\mathbf{B}^{r}(\mathbf{r})=\sum_{n=-\infty}^{\infty} r_{n} e^{i\left(q_{n} x-\kappa_{z, n}^{-} z\right)} \hat{\mathbf{y}},
$$

where an implicit time dependence of the usual form $e^{-i \omega t}$ is assumed henceforth, and where the wave vectors of the Bloch modes are defined as

$$
q_{n}=k_{x}+n G=k_{x}+n 2 \pi / L,
$$


where $G=2 \pi / L$ is the primitive vector of the reciprocal lattice. In addition, note that $\epsilon_{1} k_{0}^{2}=q_{n}^{2}+\left(\kappa_{z, n}^{-}\right)^{2}$ as determined from Maxwell's equations. Likewise, the field transmitted across the graphene grating may also be casted as a Bloch sum, reading

$$
\mathbf{B}^{t}(\mathbf{r})=\sum_{n=-\infty}^{\infty} t_{n} e^{i\left(q_{n} x+\kappa_{z, n}^{+} z\right)} \hat{\mathbf{y}}
$$

where $\left(\kappa_{z, n}^{+}\right)^{2}=\epsilon_{2} k_{0}^{2}-q_{n}^{2}$. As in the case of the incident fields, we can make use of Maxwell's curl equation $\nabla \times \mathbf{B}=$ $-i \omega \epsilon / c^{2} \mathbf{E}$ to write the corresponding reflected and transmitted electric fields from Eqs. (3) and (5). This procedure leads to

$$
\begin{aligned}
\mathbf{E}^{r}(\mathbf{r}) & =-\frac{c^{2}}{\omega \epsilon_{1}} \sum_{n=-\infty}^{\infty} r_{n}\left[\kappa_{z, n}^{-} \hat{\mathbf{x}}+q_{n} \hat{\mathbf{z}}\right] e^{i\left(q_{n} x-\kappa_{z, n}^{-} z\right)}, \\
\mathbf{E}^{t}(\mathbf{r}) & =\frac{c^{2}}{\omega \epsilon_{2}} \sum_{n=-\infty}^{\infty} t_{n}\left[\kappa_{z, n}^{+} \hat{\mathbf{x}}-q_{n} \hat{\mathbf{z}}\right] e^{i\left(q_{n} x+\kappa_{z, n}^{+} z\right)},
\end{aligned}
$$

respectively. At this stage, the coefficients $r_{n}$ and $t_{n}$ are still unknown. In order to determine them, one must impose the appropriate boundary conditions of the problem. To that end, we employ the first boundary condition stating that the $x$ component of the electric field above and below the graphene grid must be continuous, $\left.\hat{\mathbf{x}} \cdot\left(\mathbf{E}^{i}+\mathbf{E}^{r}-\mathbf{E}^{t}\right)\right|_{z=0}=0$, that is

$$
k_{z} B_{0}^{i} e^{i k_{x} x}-\sum_{n=-\infty}^{\infty} r_{n} \kappa_{z, n}^{-} e^{i q_{n} x}=\frac{\epsilon_{1}}{\epsilon_{2}} \sum_{l=-\infty}^{\infty} t_{l} \kappa_{z, l}^{+} e^{i q_{l} x} .
$$

Multiplying the previous expression with a basis function, $e^{-i q_{m} x}$, and integrating over the unit cell, yields

$$
r_{m}=\frac{k_{z}}{\kappa_{z, m}^{-}} B_{0}^{i} \delta_{m, 0}-\frac{\epsilon_{1}}{\epsilon_{2}} \frac{\kappa_{z, m}^{+}}{\kappa_{z, m}^{-}} t_{m},
$$

which links the Bloch coefficients $r_{m}$ and $t_{m}$ (and $B_{0}^{i}$ for that matter). Moreover, according to Ohm's law, the electric fields produce a current given by $\mathbf{J}=\left.\sigma(x) \hat{\mathbf{x}} \cdot \mathbf{E}^{t}\right|_{z=0} \hat{\mathbf{x}}$, which reads

$$
J_{x}(x)=\frac{\sigma(x) c^{2}}{\omega \epsilon_{2}} \sum_{n=-\infty}^{\infty} \kappa_{z, n}^{+} t_{n} e^{i q_{n} x},
$$

where $\sigma(x)$ is the position-dependent conductivity of graphene, which in the unit cell can be written as $\sigma(x)=$ $\sigma(\omega) \Theta(w / 2-|x|)$. In this expression, $\sigma(\omega)$ is the dynamical conductivity of a graphene ribbon (here assumed to be bulklike) and $\Theta(x)$ denotes the Heaviside step function [80].

We now introduce a central assumption into our analytic method, which is the validity of the edge condition [81]. This condition states that the current perpendicular to a sharp edge — such as that of a graphene ribbon-should be proportional to the square root of the distance to the edge, $\rho$; that is, $J_{x}(\rho) \propto \sqrt{\rho}$. Our assumption here is that in the regime where $k w<1$ one can interpolate the current by an expression that incorporates the edge condition at both edges of each ribbon, e.g., $x= \pm w / 2$, simultaneously. Therefore, this ansatz allows us to write the current within a ribbon in the unit cell as

$$
J_{x}(x)=\chi e^{i k_{x} x} \sqrt{w^{2} / 4-x^{2}} \Theta(w / 2-|x|),
$$

where $\chi$ is a coefficient to be determined. As a first step towards the determination of the coefficient $\chi$, we now argue that Eqs. (10) and (11) must give rise to the same induced current (since they represent the same physical quantity). Hence, one may write the relation (in the unit cell)

$\chi e^{i k_{x} x} \sqrt{w^{2} / 4-x^{2}} \Theta(w / 2-|x|)=\frac{\sigma(x) c^{2}}{\omega \epsilon_{2}} \sum_{n=-\infty}^{\infty} \kappa_{z, n}^{+} t_{n} e^{i q_{n} x}$,

which, after multiplying by a basis function, $e^{-i q_{m} x}$, and integrating over the unit cell, produces

$\chi \frac{L}{4 m} J_{1}(m \pi w / L)=\frac{\sigma(\omega) c^{2}}{\omega \epsilon_{2}} \sum_{n=-\infty}^{\infty} \kappa_{z, n}^{+} t_{n} \frac{\sin ([n-m] \pi w / L)}{[n-m] \pi w / L}$,

where $J_{1}(x)$ is the first-order Bessel function of the first kind [80]. This expression defines the coefficient $\chi$ in terms of the Bloch amplitudes $t_{m}$, and whose combination with Eq. (9) connects the coefficients $\chi, r_{m}$, and $t_{m}$. In order to close the system of equations, we require another expression relating these quantities. Such an "extra" equation is the other boundary condition holding for this system; in particular, the discontinuity of the magnetic field across the graphene grid due to the presence of the surface current induced by the electric field, i.e., $\hat{\mathbf{z}} \times\left.\left(\mathbf{B}^{t}-\mathbf{B}^{r}-\mathbf{B}^{i}\right)\right|_{z=0}=\mu_{0} J_{x} \hat{\mathbf{x}}$ which, after applying the same operations that led to Eqs. (9) and (13), gives

$$
B_{0}^{i} \delta_{m, 0}=t_{m}-r_{m}+\mu_{0} \chi \frac{w}{4 m} J_{1}(m \pi w / L),
$$

thereby closing the system. Finally, the combination of Eqs. (9) and (14) allows us to write the $t_{m}$ 's as

$$
t_{m}=\frac{\epsilon_{2} \kappa_{z, m}^{-}}{\epsilon_{1} \kappa_{z, m}^{+}+\epsilon_{2} \kappa_{z, m}^{-}}\left[2 B_{0}^{i} \delta_{m, 0}-\mu_{0} \chi \frac{w}{4 m} J_{1}(m \pi w / L)\right],
$$

which, after using Eq. (13), endows us an expression for the coefficient $\chi$ (from which the Bloch amplitudes $t_{m}$ and $r_{m}$ directly follow); that is

$$
\chi=\frac{2 \kappa_{z, 0}^{+} \kappa_{z, 0}^{-}}{\epsilon_{1} \kappa_{z, 0}^{+}+\epsilon_{2} \kappa_{z, 0}^{-}} \frac{\sigma(\omega) c^{2}}{\omega} \frac{B_{0}^{i}}{\Lambda(\omega)},
$$

where the quantity $\Lambda(\omega)$ is defined as

$\Lambda(\omega)=\frac{w}{4} \sum_{n=-\infty}^{\infty} \frac{1}{n} J_{1}(n \pi w / L)\left[1+\frac{\sigma(\omega)}{\omega \epsilon_{0}} \frac{\kappa_{z, n}^{+} \kappa_{z, n}^{-}}{\epsilon_{1} \kappa_{z, n}^{+}+\epsilon_{2} \kappa_{z, n}^{-}}\right]$.

It should be stressed that the sum in the previous expression needs to be judiciously evaluated, since it is a sum with alternating signs (check Appendix A 1 for details).

Therefore, Eqs. (9) and (15)-(17) provide us with complete knowledge of the electromagnetic scattering and subsequent excitation of graphene plasmons within the ribbons which make up the periodic system. 


\section{B. Transmittance, reflectance, and absorbance for normal incidence}

Here we consider the particular case where the impinging radiation strikes the graphene grid at normal incidence (see Appendix A 2 for oblique incidence), for which we have $k_{x}=0$ and $k_{z}=\sqrt{\epsilon_{1}} k_{0}$, so that $q_{n}=n G$. In addition we remark that here, as in most experimental configurations, the $z$ component of the scattered wave vectors remains real only for the zeroth mode, i.e., $\kappa_{z, 0}^{+/-}=\sqrt{\epsilon_{2 / 1}} k_{0}$, while for the other diffraction orders it is imaginary, that is $\kappa_{z, n}^{+/-}=$ $i \sqrt{q_{n}^{2}-\epsilon_{2 / 1} k_{0}^{2}}$. The reason for this is that often the period of the grating is much smaller than the impinging wavelength, $L \ll \lambda$, and thus $q_{n}^{2} \gg \epsilon_{2 / 1} k_{0}^{2}, \forall n \neq 0$. This is no coincidence, since our goal is to surpass the momentum imbalance between the incident light and GSPs. This can only be effectively achieved by fabricating subwavelength gratings. Therefore, we take $q_{n}>\sqrt{\max \left(\epsilon_{1}, \epsilon_{2}\right)} k_{0}$ for $n \neq 0$ henceforth. Consequently, only the zeroth mode reaches the far field.

In possession of Eqs. (9) and (15)-(17), we have all the necessary ingredients to compute the scattering efficiencies for EM radiation striking the array of graphene ribbons. From the aforementioned expressions, the reflectance, transmittance, and absorbance by the graphene grid read (see Appendix A 2 for a detailed derivation)

$$
\begin{gathered}
R(\omega)=\left|1-\frac{2 \sqrt{\epsilon_{1}}}{\sqrt{\epsilon_{1}}+\sqrt{\epsilon_{2}}}+\mu_{0} \frac{\chi}{B_{0}^{i}} \frac{\pi w^{2}}{8 L} \frac{\sqrt{\epsilon_{1}}}{\sqrt{\epsilon_{1}}+\sqrt{\epsilon_{2}}}\right|^{2}, \\
T(\omega)=\frac{\operatorname{Re}\left\{1 / \sqrt{\epsilon_{2}}\right\}}{\operatorname{Re}\left\{1 / \sqrt{\epsilon_{1}}\right\}}\left|\frac{\sqrt{\epsilon_{2}}}{\sqrt{\epsilon_{1}}+\sqrt{\epsilon_{2}}}\left(2-\mu_{0} \frac{\chi}{B_{0}^{i}} \frac{\pi w^{2}}{8 L}\right)\right|^{2}, \\
A(\omega)=1-R(\omega)-T(\omega),
\end{gathered}
$$

respectively [and recall Eqs. (16) and (17) for $\chi$ ]. From the inspection of the above equations it is clear that the plasmonic resonances are controlled by the poles of $\chi$ [or, similarly, $\left.-\operatorname{Im}\left\{\Lambda^{-1}(\omega)\right\}\right]$. Analyzing carefully the structure of the quantity $\Lambda(\omega)$, we readily identify that these occur whenever the condition

$$
\frac{\epsilon_{1}}{\sqrt{(n G)^{2}-\epsilon_{1} k_{0}^{2}}}+\frac{\epsilon_{2}}{\sqrt{(n G)^{2}-\epsilon_{2} k_{0}^{2}}}+i \frac{\sigma(\omega)}{\omega \epsilon_{0}}=0
$$

weighted by the factor $J_{1}(n \pi w / L) / n$ is met. Notice that Eq. (21) is nothing but the implicit expression for the dispersion relation of GSPs [18-20,22,24] with wave vector $q_{n}=n G$. However, what particular Bragg modes constitute the leading contributions for GSP excitation strongly depends on the filling ratio $w / L$ (please refer to Appendix A 3 for further details).

\section{RESULTS AND DISCUSSION}

\section{A. Signatures of graphene plasmon resonances}

Having formulated our analytical model, we are now able to compute the absorbance, reflectance and transmittance spectra of EM radiation impinging on a periodic grid of graphene ribbons at normal incidence. Therefore, the results presented below are based on the outcome of Eqs. (18)-(20). In the
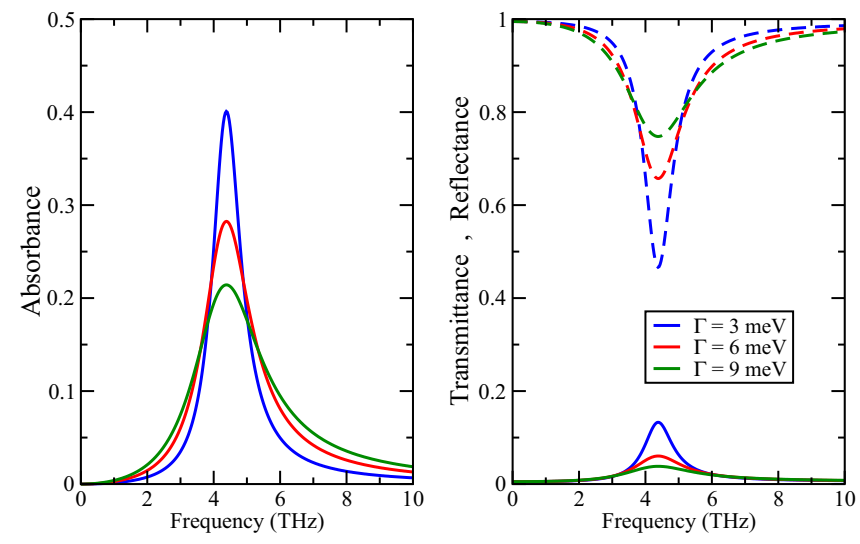

FIG. 2. Absorbance spectra (left panel) as well as transmittance and reflectance spectra (dashed and solid lines in right panel, respectively) of a $p$-polarized plane wave impinging on a periodic grid of graphene ribbons for varying values of $\Gamma=\hbar \gamma$. The remaining parameters are $E_{F}=0.45 \mathrm{eV}, w=2 \mu \mathrm{m}, L=4 \mu \mathrm{m}, \epsilon_{1}=3, \epsilon_{2}=4$, and $\theta=0$ (normal incidence).

following, we take the conductivity of the graphene ribbons as the Drude conductivity of bulk graphene (see Appendix B). This is a rather good approximation for doped graphene ribbons in the $\mathrm{THz}$ spectral range, as long as the ribbons are not too small (e.g., wider than several tens of nanometers [78,79]). In particular, in Fig. 2 we show the absorbance spectra (left panel) and corresponding reflectance and transmittance spectra (right panel) for different values of the damping parameter, $\Gamma$. The main feature figuring in the various spectra is the presence of a well-defined peak in absorption signaling the excitation of graphene plasmons. This GSP-assisted effect yields a dramatic enhancement in the absorbance spectra of the grating, owing to the coupling of free-propagating $\mathrm{THz}$ radiation to plasmons supported by the graphene ribbons which compose the periodic grid. Note that the aforementioned GSP-induced absoption comes hand in hand with a suppression in transmittance and with an increase in reflectance at the GSP resonant frequencies, which roughly correspond to the poles of $\chi$ (or, in other words, the zeros of $\Lambda$ ). Without surprise, smaller values of $\Gamma$ render sharper resonances, with these becoming successively broader and less pronounced as the electronic scattering rate increases. Also, the resonance frequency of the GSPs modes depends weakly on the value of $\Gamma$, as it remains essentially unchanged despite the different values of the damping parameter.

It should be stressed that, in principle, the interaction of EM radiation with the grating gives rise to multiple plasmon resonances with $q \approx(2 m+1) \pi / w$ (for $m=$ $0,1,2, \ldots)[24,49,82]$. The most prominent resonance corresponds to the fundamental plasmon mode, while the higherorder resonances become increasingly weaker. Note that in the present model the latter are necessarily ignored, owing to the choice of ansatz for the current [cf. Eq. (11)] which can only account for the dipole-like fundamental resonance (the one that appears in Fig. 2). Fortunately, this resonance carries most of the spectral weight (see Appendix A4) and it clearly dominates the polaritonic spectrum [22,24]; in fact, the resonances that emerge at higher frequencies are often invisible (or barely visible) in many experiments, since they can only be detected for small values of $\Gamma$. 

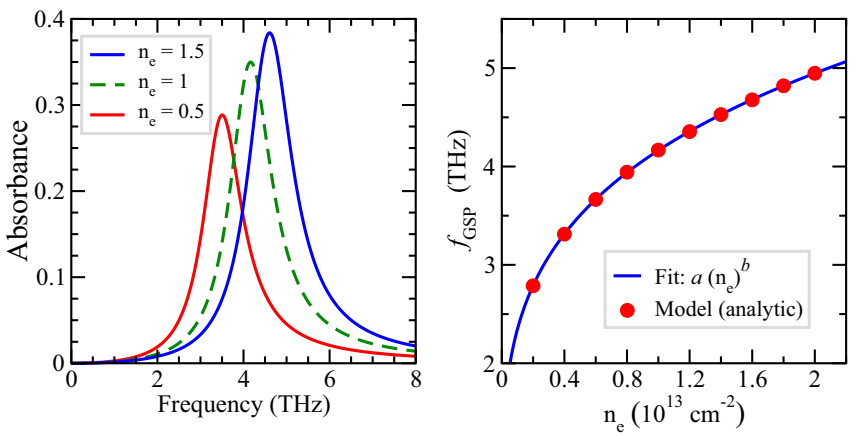

FIG. 3. Dependence of the GSP frequency on the electronic density of the graphene ribbons. Left panel: absorbance spectra for different selected values of $n_{e}$; the legend gives $n_{e}$ in units of $10^{13} \mathrm{~cm}^{-2}$. Right panel: resonant frequencies-retrieved from our analytic theory (points) - for several values of $n_{e}$, and corresponding fitting function to those points; that is, $f_{\mathrm{GSP}}\left(n_{e}\right) \propto n_{e}^{b}$ with $b=$ $0.249 \simeq 1 / 4$, in accordance with what is expected for graphene plasmons (see main text). Parameters: $\Gamma=3.7 \mathrm{meV}, w=2 \mu \mathrm{m}$, $L=4 \mu \mathrm{m}, \epsilon_{1}=3, \epsilon_{2}=4$, and $\theta=0$ (normal incidence). We have used $E_{F}=\hbar v_{F} \sqrt{\pi n_{e}}$, with $v_{F} \approx 1.1 \times 10^{6} \mathrm{~m} / \mathrm{s}$.

We now explore the dependence of the GSP-induced absorption spectra on the different parameters of the system. One of the most important parameters is the electronic density, $n_{e}$. This quantity is related to the material's Fermi energy via $E_{F}=\hbar v_{F} \sqrt{\pi n_{e}}$, where $v_{F} \approx 1.1 \times 10^{6} \mathrm{~m} / \mathrm{s}$ is the Fermi velocity of the Dirac fermions in graphene [83,84]. The density of graphene charge carriers can be easily controlled by means of electrostatic gating. This possibility is of extreme relevance in graphene plasmonics, since it enables the excitation and control of tunable GSPs with tailored properties at the distance of a voltage knob. The effect of varying the electronic density within the graphene ribbons which constitute the grating is demonstrated in Fig. 3. From the figure, it is clear that GSP resonances become stronger and shift toward higher frequencies as the density of charge carriers increases. In order to quantify such behavior, in the right panel of Fig. 3 we have plotted the GSP frequency (corresponding to the fundamental mode) as a function of the doping level, to which we have fitted a function of the type $f_{\mathrm{GSP}}\left(n_{e}\right) \propto n_{e}^{b}$, having obtained $b=0.249 \simeq 1 / 4$ for the exponent (fitting parameter). ${ }^{1}$ This therefore demonstrates that the observed resonances scale with the electronic density as $f_{\mathrm{GSP}} \propto n_{e}^{1 / 4}$, which is a specific signature of graphene plasmons [22,24,48,49]. In contrast, in typical 2DEGs a scaling with $n_{e}^{1 / 2}$ is observed instead. The different scaling for graphene is a direct consequence of the linear dispersion exhibited by the Dirac particles in this material $[12,13]$.

An alternative way to tune the GSP resonances is to pattern grids of graphene ribbons of different widths, $w$. For the sake of clarity, we shall keep the filling ratio $w / L=1 / 2$ constant. Figure 4 depicts the calculated absorbance spectra for periodic arrays of graphene ribbons of different widths. Notice that structures with narrower ribbons yield GSPs with

${ }^{1}$ The fitting function was obtained using the least-squares method.

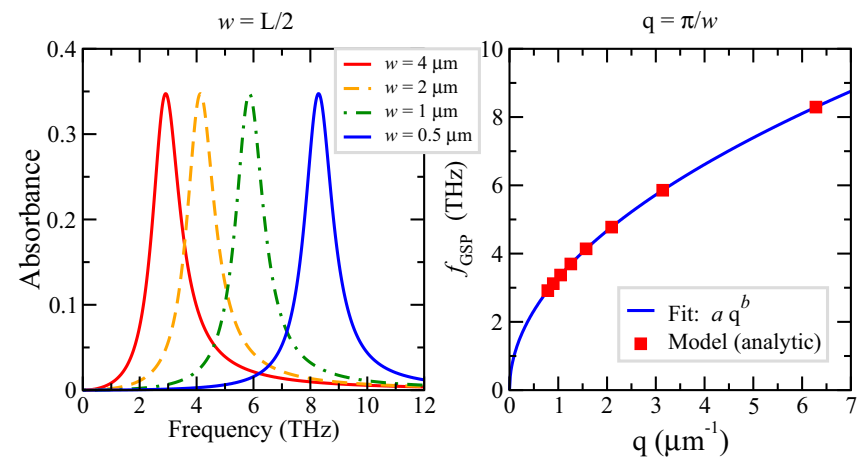

FIG. 4. Dependence of the GSP frequency on the period of the graphene grating, $L$. We kept the ratio $w=L / 2$ fixed, and therefore the fundamental GSP mode carries a wave vector of about $q \sim \pi / w$. In the right panel we show the position of the GSP resonances rendered by the analytical method incorporating the edge condition as a function of the GSP wave vector (square data points). To these data we have fitted a curve $f_{\mathrm{GSP}}(q) \propto q^{b}$, having obtained a exponent $b=0.502 \sim 1 / 2$. Parameters: $E_{F}=0.4 \mathrm{eV}, \Gamma=3.7 \mathrm{meV}, \epsilon_{1}=3$, $\epsilon_{2}=4$, and $\theta=0$.

higher energies. The fundamental plasmonic resonance of the grating resembles the excitation of a GSP in extended graphene with $q \sim \pi / w$. Such large wave vectors can only be attained due to the contribution of the several Bragg diffraction orders originating from the interaction of the incident light with the grid. Within this reasoning, Fig. 4 shows that the plasmonic resonances scale as $\sqrt{q}$, in a similar way that plasmons in an unpatterned, continuous graphene sheet do. We have also found that, while the filling ratio $w / L$ has a significant impact on the position of the plasmonic resonance of the graphene grid, the physics of the system is largely determined by the ribbon size, i.e. $f_{\mathrm{GSP}} \propto w^{-1 / 2}$ provided that $w / L<1 / 2$; that is, that the interaction between neighboring ribbons is small.

The calculated electric field akin to the graphene plasmons supported by the ribbons which constitute the grating is depicted in Fig. 5 (depicting the unit cell of a representative array of ribbons with dimensions $w=2 \mu \mathrm{m}$ and $L=4 \mu \mathrm{m}$ ). Such computation is straightforward once the resonant frequency akin to the fundamental GSP mode is determined; that information is then fed into the Bloch amplitudes (9) and (15) that represent the scattered field. The figure plainly demonstrates the potential graphene plasmons have to squeeze EM fields into deep subwavelength dimensions. Notice that most of the modal energy is concentrated in the immediate vicinity of the graphene ribbons. In addition, the spatial distribution of the electric field is not uniform along the ribbons' transverse direction: the density of charge carriers (and thus the electric field) is higher at both edges of the ribbons. The charge density is also antisymmetric with respect to the ribbons' midpoint, bearing some similarity to an electric dipole. Such extreme field localization plays a pivotal role, for instance, in biosensing, allowing the detection of minute variations in the local dielectric environment due to the presence or adsorption of a given target analyte. This property, together with ability to tune the GSP resonances, enables not only unprecedentedly large field overlaps, but also provides a route to tailor the interaction of GSPs with the vibrational 


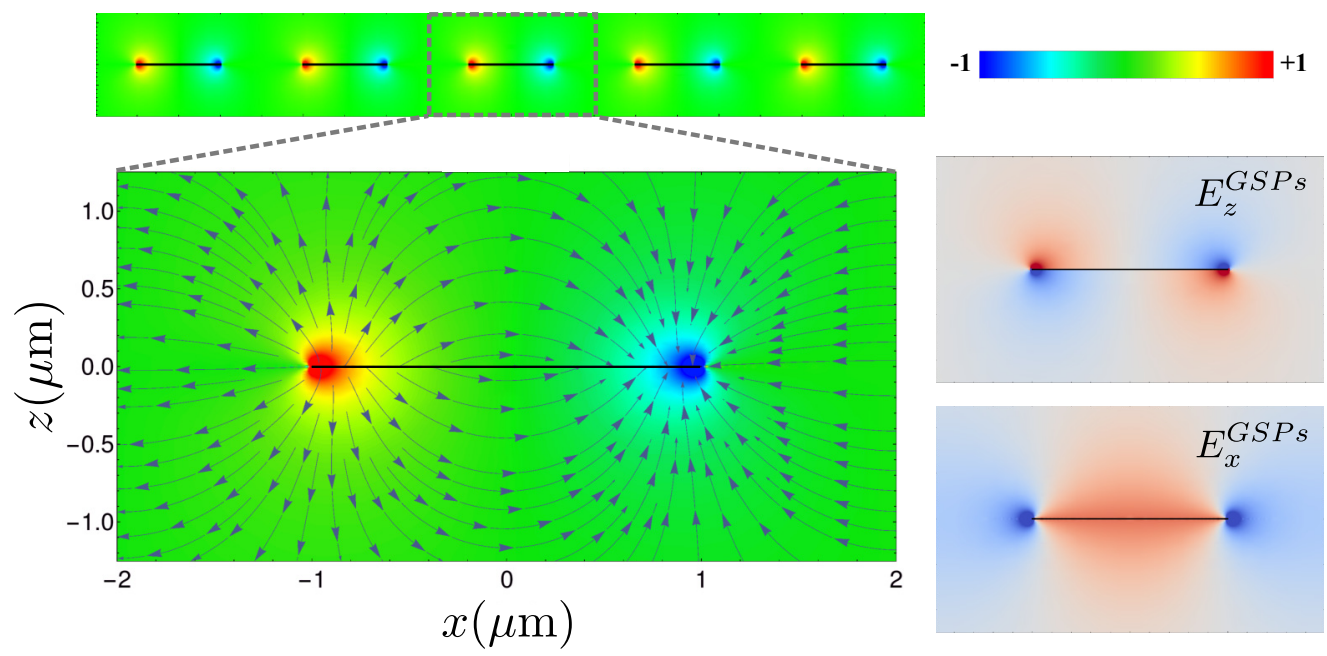

FIG. 5. Electric field representing graphene plasmons excited in the graphene ribbons which compose the grid (with dimensions $w=2 \mu \mathrm{m}$ and $L=4 \mu \mathrm{m})$. The figures show the plasmonic fields in the system's unit cell, and the graphene ribbon is indicated by the horizontal black line. Left panel: vectorial representation of the electric field (in the $y=0$ plane) due to GSPs, $\mathbf{E}^{\mathrm{GSPs}}(x, z)=E_{x}^{\mathrm{GSPs}}(x, z) \hat{\mathbf{\mathbf { x }}}+E_{z}^{\mathrm{GSPs}}(x, z) \hat{\mathbf{z}}$. The intensity plot refers to the quantity $\operatorname{sgn}(z) E_{z}^{\mathrm{GSP}}$ which roughly highlights the charge density within each graphene ribbon. Righ panel: normalized spatial distributions of the electric field components $E_{z}^{\mathrm{GSPs}}$ (top) and $E_{x}^{\mathrm{GSPs}}$ (bottom). The spatial range covered in these subpanels is the same as in the main panel. We note that only modes corresponding to plasmonic (evanescent) modes were included in the sums figuring in the Bloch expansions, which for the parameters used here encompass all modes with the exception of the specular one (i.e., with $n=0$ ). Parameters: $E_{F}=0.4 \mathrm{eV}, \Gamma=3.7 \mathrm{meV}$, and $\epsilon_{1}=\epsilon_{2}=4$.

resonances of biochemical molecules, thereby achieving huge spectral overlaps that allow specific label-free detection of biomolecules via their vibrational fingerprints [26,27].

We further note that the overall spatial configuration of the field illustrated in Fig. 5 is qualitatively maintained throughout a wide range of ribbon widths, from the micrometer to the nanometer size, provided that we are within the quasistatic regime (i.e., $q_{\mathrm{GSP}} \gg k_{0}$ ) and at resonant frequencies below $E_{F} / \hbar$, beyond which GSPs become quenched owing to the onset of interband Landau damping [18,24]. This scale invariance is a property of the electrostatic limit $[18,24,85]$. Note, however, that we include retardation in our calculations nevertheless.

The results presented above, covering an appreciable vast parameter space, suggest that our analytical model is able to correctly describe the fundamental plasmonic excitations which arise in periodic grids of graphene ribbons. We thus have built an analytic framework which delivers closed-form expressions for the spectra which can be easily evaluated, and that yield results consistent with those found in the literature [18-20,22,24,48,49].

\section{B. THz plasmons in graphene microribbons}

In order to determine to what extent our analytical model is capable of explaining experimental spectra, we shall test our theory against measured data taken from the experiments performed by Ju et al. [48]. To that end, we mimic the experimental setup by feeding the reported empirical parameters into our equations. In addition, we concurrently employ a semianalytical technique introduced elsewhere [22,24,75-77] which represents the conductivity of graphene (and resultant current) in terms of a Fourier series (see Appendix C). This has the advantage of taking into account not only the fundamental plasmonic resonance, but also the higher-order ones. On the other hand, it requires the numerical solution of a linear algebra problem and therefore we refer to it as a semianalytical method hereafter. We further emphasize that, to the best of our knowledge, so far no attempt has been made to perform a direct comparison of the outcome of this latter method against available experimental data. Nevertheless, it is still far less computationally demanding than fully numerical simulations such as the finite-difference time-domain (FDTD) or finite element method (FEM) techniques [86].

In their experiments, the authors of Ref. [48] fabricated three different samples containing periodic grids of graphene ribbons with widths of 4,2 , and $1 \mu \mathrm{m}$, while maintaining the ratio $L=2 w$ unchanged. Moreover, the authors have concluded that an effective dielectric constant of $\epsilon_{\text {eff }}=5$ adequately accounts for the intricate optical constants of the cladding dielectrics (ion gel and $\mathrm{SiO}_{2} / \mathrm{Si}$ ), and have reported a scattering rate of $\gamma /(2 \pi)=4 \mathrm{THz}$ [48]. While at $\mathrm{THz}$ frequencies the conductivity of graphene can be approximated by its Drude expression, here we model the conductivity as obtained using the Kubo formula at room temperature (see Appendix B). This is necessary here because the experimental data of Ju et al. [48] refer to the change in transmittance with respect to the same quantity measured at the "charge neutral point" (CNP) (when the Fermi level is at the so-called Dirac point) where neither finite-temperature nor interband processes can be neglected.

The comparison between the calculated GSP-induced (normalized) change in transmittance, $-\Delta T=T_{\mathrm{CNP}}-T$, and the experimental data is portrayed in Fig. 6. The observed agreement between theory and experiment is outstanding and constitutes compelling evidence that both theories are capable of interpreting the measured spectra. The peaks visible in 


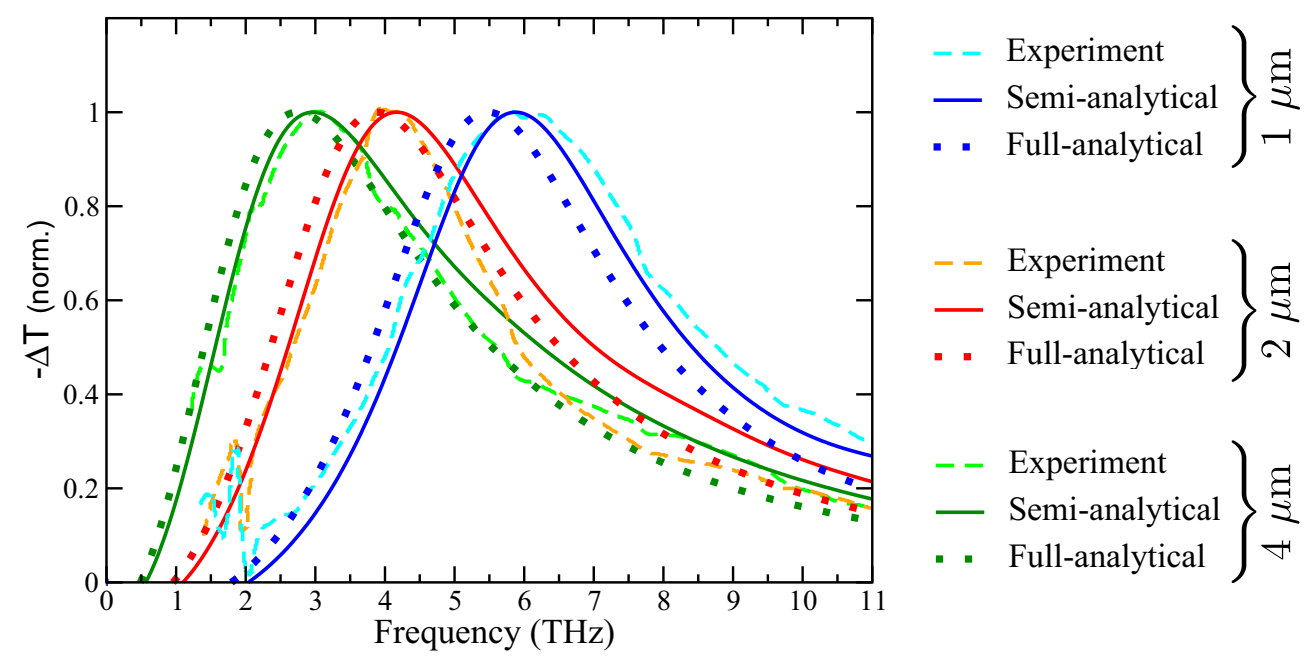

FIG. 6. Normalized plasmon-induced change in transmittance relative to the CNP, $-\Delta T=T_{\mathrm{CNP}}-T$, in periodic grids of graphene ribbons with different dimensions: $w=4 \mu \mathrm{m}$ (green), $w=2 \mu \mathrm{m}$ (red), $w=1 \mu \mathrm{m}$ (blue), and $w / L=1 / 2$ throughout. The dashed lines correspond to experimentally measured spectra [48], while the dotted lines and solid lines correspond to the spectra obtained using our full-analytical model and the semianalytical technique (see Appendix C), respectively. We have used the following parameters, in accordance with Ref. [48]: $E_{F}=0.497 \mathrm{eV}, \Gamma=16.5 \mathrm{meV}$, and $\epsilon_{1}=\epsilon_{2}=5$.

the figure originate from the excitation of the main GSP plasmonic resonance supported by the graphene ribbons which form the grid. The shifting of the plasmon resonances towards higher frequencies as a consequence of the narrowing of the ribbons exhibits the predicted $f_{\mathrm{GSP}} \propto w^{-1 / 2}$ scaling behavior. Although the degree of accordance between the data and the full-analytical model is rather good, it seems that this model slightly underestimates the resonant frequency. On the other hand, the agreement among the experimental measurements and the spectra obtained by the semianalytical model is indeed quite remarkable, with the computed (measured) GSP resonances located at 2.9 (3), 4 (4.1), and 5.6 (6) $\mathrm{THz}$ for arrays with ribbon widths of $4,2 \mathrm{~m}$ and $1 \mu \mathrm{m}$, respectively. Therefore, a possible motif for the small redshift visible in the spectra produced by the full-analytic theory may be concerned either with the fact that it neglects the higher-order plasmon resonances and/or with the approximation made for the current involving the edge condition; for instance, it may not be exactly modeled by a square root as in Eq. (11) (see Appendix A 5). Still, and despite this fact, the fidelity of the analytical model remains very good.

It should be appreciated that the use of either one of the above-mentioned analytical or semianalytical techniques, apart from requiring less resources, provide a level of physical insight and intuition that numerical methodologies based on the numerical solution of Maxwell's equations simply cannot envision.

\section{Hybrid mid-IR plasmons in graphene nanoribbons: Plasmon-phonon coupling}

Graphene plasmonics has the potential to become a viable tool for nanophotonic devices working within a broad spectral window, from the THz/far-IR up to mid-IR frequencies. Remarkably, routes to bring graphene plasmonics to near-IR and visible frequencies have already been proposed from a theoretical perspective [18].
At the time of writing, many experiments have reported GSPs at mid-IR frequencies [26,27,52,55-57,60,61]. Coupling light to graphene plasmons at those frequencies can be realized in nanostructured graphene with typical dimensions from several tens of nanometers to a few hundred nanometers. The mid-IR spectral range is a particularly important one, as many biological and chemical compounds exhibit resonances in that region of the EM spectrum. Thus, tunable graphene plasmons may be perceived as fertile ground for applications in biochemical sensing and spectroscopy. Furthermore, when graphene is deposited in a polar substrate-such as hexagonal boron nitride $(\mathrm{hBN})$ or $\mathrm{SiO}_{2}$ - the Fuchs-Kliewer SO phonons [87] of the substrate can couple to plasmons in graphene via Fröhlich interaction [88], leading to the emergence of new hybrid modes dubbed graphene surface plasmon-phonon polaritons [49,61] (GSPPhs). In order to account for the optical phonons arising in the neighboring polar material(s), their corresponding frequency-dependent, complex-valued dielectric function(s) can be modeled using adequate Lorentz oscillator models [89] incorporating the phononic resonances, or more evolved models, e.g., based on Gaussian functions and integrals [90]. The hallmark of strong coupling between graphene plasmons and SO phonons is the complete reshaping of the traditional $f_{\mathrm{GSP}} \propto \sqrt{q}$ dispersion of bare GSPs into a set of multiple well-defined branches ascribed to hybrid GSPPhs modes possessing mixed plasmonic and phononic character, as demonstrated in Fig. 7(c) for extended graphene sitting on $\mathrm{SiO}_{2}$ (see caption for further details). In particular, notice the evident anti-crossing behavior of the plasmon-phonon bands in the vicinity of the SO frequencies.

In Figs. 7(a) and 7(b) we compare the results for arrays of graphene ribbons obtained using the analytic [panel (a)] and semianalytic [panel (b)] methods (solid lines) against the experimental spectra (light-brown points) collected by Luxmoore et al. [52] Their data show evidence of strong interaction of GSPs-excited in periodic grids of graphene 

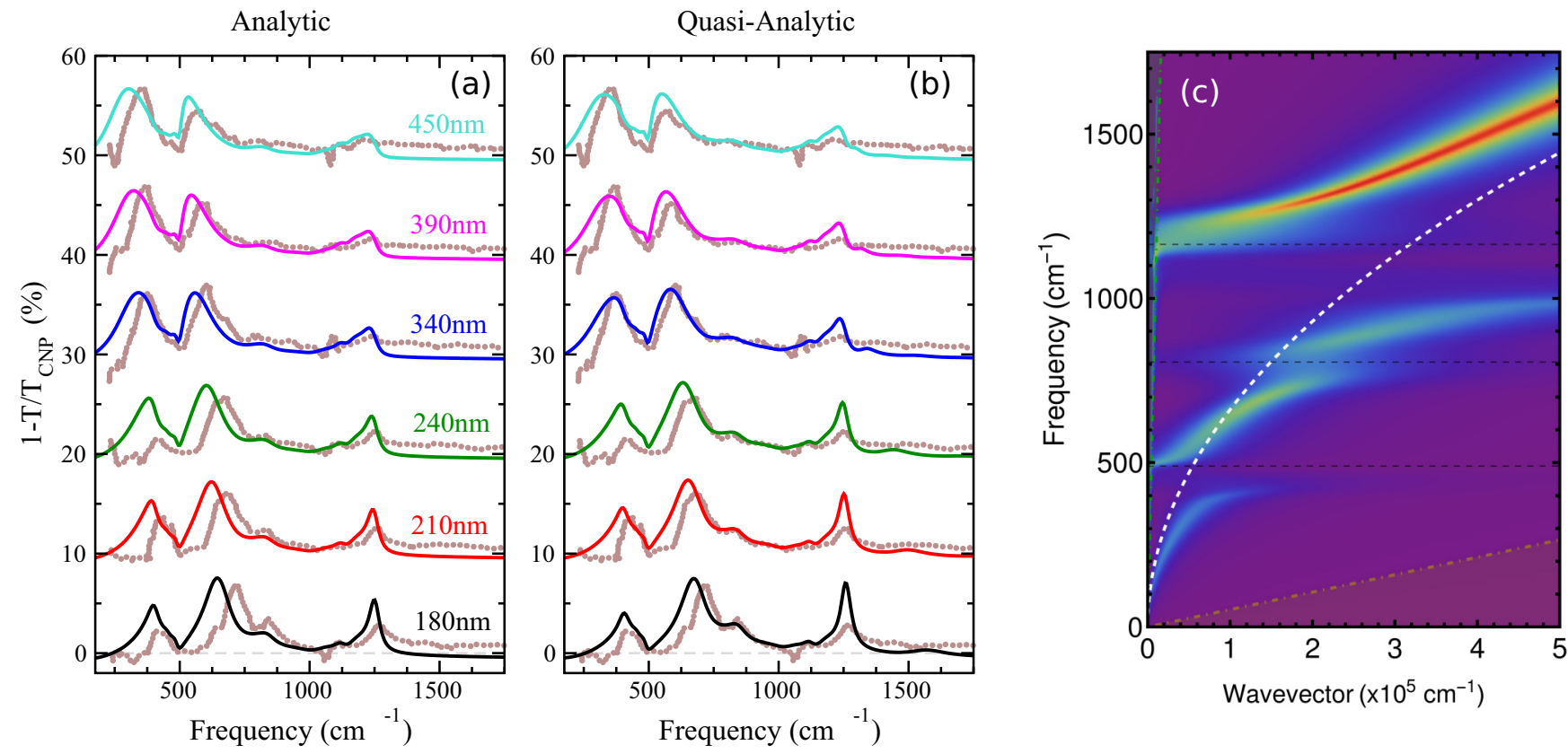

FIG. 7. Hybrid mid-IR graphene plasmon-phonon polaritons excited in period gratings of graphene nanoribbons sitting on a polar $\left(\mathrm{SiO}_{2}\right)$ substrate. Experimental data (light-brown points) and corresponding extinction spectra calculated using (a) the analytical model and (b) the semi-analytical model (solid lines). The computations were carried out in accordance with the experimental parameters [52] $E_{F}=0.37 \mathrm{eV}$, $\theta=0$ (normal incidence), and $\epsilon_{1}=1$; the dielectric function of $\mathrm{SiO}_{2}, \epsilon_{2} \equiv \epsilon_{\mathrm{SiO}_{2}}(\omega)$, was taken from the literature [90]. We have used electronic scattering rates corresponding to about 25-30 meV depending on the sample. (c) Loss function (via Im $r_{\mathrm{TM}}$ ) for extended graphene deposited on $\mathrm{SiO}_{2}$, with the uncoupled GSP spectrum superimposed (white dashed line); we note that this serves only as an eye guide to the interpretation of the data, since we expect the polaritonic spectrum akin to the periodic grid of nanoribbons to be slightly different from that of unpatterned, extended graphene.

nanoribbons-with the three SO phonons of the underlying $\mathrm{SiO}_{2}$ substrate. In our modeling, we have met the experimental configuration of the fabricated devices, consisting of doped $\left(E_{F}=0.37 \mathrm{eV}\right)$ nanoribbons with widths ranging from $450 \mathrm{~nm}$ down to $180 \mathrm{~nm}$ arranged in a periodic array with periodicity $L=5 w / 2$ [52]. The dynamical dielectric function of the $\mathrm{SiO}_{2}$ substrate was taken from the literature [90], and we have employed, as before, the optical conductivity of graphene under Kubo's framework.

It is worth to highlight that both the analytic and semianalytic theories outlined above fit admirably to the experimental data, whose structure is now much more intricate than the one seen for microribbons in the $\mathrm{THz}$ range. The GSPPh-induced extinction spectrum $\left(1-T / T_{\mathrm{CNP}}\right)$ of the several samples presented in Fig. 7 reveals the existence of multiple peaks, which correspond to the four polaritonic bands visible in the figure's last panel. We stress that, as expected, all four resonances shift toward higher frequencies upon decreasing ribbon size. Note, however, that they disperse at different rates. This is a direct consequence of the relative plasmon-to-phonon content which tends to vary depending on the distance each resonance is from the SO flat bands: the more plasmon-like the hybrid GSPPhs modes are (i.e., the farther they are from the uncoupled SO bands), the faster they disperse. Another key element, recognizable in the spectra, is the clear transfer of spectral weight from the first peak to the other resonances ascribed to higher GSPPhs bands. Together, the abovementioned features constitute unambiguous manifestations of anticrossing behavior.
From the first two panels of Fig. 7 it is apparent that both models closely follow the experimental data, thereby confirming their adequacy to describe the empirical extinction spectra. As in the case of the microribbons, the fully analytical method tends to underestimate slightly the position of the resonances, while the semianalytical method yields an excellent agreement, particularly for the samples with wider ribbons. The small deviation from the data observed in the spectra of the narrower ribbons may have multiple origins exogenous to our theory; for instance, an incomplete knowledge of the dielectric properties of the particular $\mathrm{SiO}_{2}$ substrate used in the experiments and/or the effect of edge damage (and defects) introduced during the etching process [49,52,91]-which, naturally, should be more pronounced for smaller ribbons, and may yield ribbon edges with impaired electrical activity (therefore rendering effective widths smaller than the actual ribbon widths [49]). Here, we neglect the impact of the latter since it has been shown that the damage at the edges of each ribbon is highly heterogeneous [52,91].

The theoretical results produced in this work and subsequent confrontation against experimental data confirm the ability of the theoretical tools developed here to simulate and interpret spectra taken from real-world experiments, providing excellent, reliable results almost instantaneously ${ }^{2}$ without the need of substantial computational resources.

\footnotetext{
${ }^{2}$ Using a standard personal laptop equipped with a dual-core (four threads) $2.30 \mathrm{GHz}$ processor.
} 


\section{CONCLUSIONS}

In conclusion, we have developed a novel analytical approach, based on the edge condition and Bloch expansions for the fields, to describe graphene plasmons excited in periodic grids of graphene ribbons. We solved the scattering problem and provided simple closed-form expressions to compute the reflectance, absorbance, transmittance, and related extinction spectra. We then benchmarked the results of our analytical theory using a semianalytical model, and tested both techniques against experimental data available in the literature $[48,52]$. Our results show a very good agreement between the theoretical curves and the empirical data, which constitutes compelling evidence for the validity of the aforementioned theories. That concordance extends from the $\mathrm{THz}$, using microribbon arrays, to the mid-IR spectral region, using nanoribbons. In the latter domain, we have also investigated hybrid GSPPhs excitations that arise from the interaction of GSPs with the $\mathrm{SO}$ phonons of the $\mathrm{SiO}_{2}$ substrate, leading to the appearance of four composite modes featuring spectral weight transfer, which is indicative of anticrossing behavior (resulting from the reconstruction of the bare GSPs spectrum owing the polar coupling).

The approaches developed in this work have two main advantages: (i) They endow us with a deeper insight and sense of the physics governing plasmonic excitations in engineered graphene structures and (ii) they render viable simulations of experimentally relevant quantities, on-demand and almost instantaneously, without the cost of lengthy, computationally demanding full-wave numerical packages, at least for patterned structures with a fair degree of symmetry. On the other hand, these naturally cannot compete with fully numerical techniques such as FEM simulations, in terms of versatility when dealing with many different and complex geometries.

Our findings suggest that both the analytical and the semianalytical models described here could be used to develop new forefront nanophotonic experiments based on graphene plasmonics, which is emerging as a promising field to deliver cutting-edge optoelectronic devices with tailored light-matter interactions.

\section{ACKNOWLEDGMENTS}

The authors thank N. Asger Mortensen for insightful and valuable comments. P.A.D.G. acknowledges financial support from Fundação para a Ciência e a Tecnologia (Portugal) via Grant No. PD/BI/114376/2016. N.M.R.P. and Y.V.B. acknowledge financial support from the European Commission through the project "Graphene-Driven Revolutions in ICT and Beyond" (Ref. No. 696656). This work was partially supported by the Portuguese Foundation for Science and Technology (FCT) in the framework of the Strategic Financing project UID/FIS/04650/2013. The Center for Nanostructured Graphene is sponsored by the Danish National Research Foundation, Project DNRF103.

\section{APPENDIX A}

\section{Convergence of the sum in $\Lambda(\omega)$}

Notice that our results for the reflection and transmission amplitudes fundamentally depend on $\chi$, which in turn strongly depends on the function $\Lambda(\omega)$. The latter reads [cf. Eq. (17)]

$$
\begin{aligned}
\Lambda(\omega)= & \frac{w}{4} \sum_{n=-\infty}^{\infty} \frac{1}{n} J_{1}(n \pi w / L)\left[1+\frac{\sigma(\omega)}{\omega \epsilon_{0}} \frac{\kappa_{z, n}^{+} \kappa_{z, n}^{-}}{\epsilon_{1} \kappa_{z, n}^{+}+\epsilon_{2} \kappa_{z, n}^{-}}\right] \\
= & \frac{\pi w^{2}}{8 L}\left[1+\frac{\sigma(\omega)}{\omega \epsilon_{0}} \frac{\kappa_{z, 0}^{+} \kappa_{z, 0}^{-}}{\epsilon_{1} \kappa_{z, 0}^{+}+\epsilon_{2} \kappa_{z, 0}^{-}}\right] \\
& +\frac{w}{2} \sum_{n=1}^{N} \frac{1}{n} J_{1}(n \pi w / L)\left[1+\frac{\sigma(\omega)}{\omega \epsilon_{0}} \frac{\kappa_{z, n}^{+} \kappa_{z, n}^{-}}{\epsilon_{1} \kappa_{z, n}^{+}+\epsilon_{2} \kappa_{z, n}^{-}}\right],
\end{aligned}
$$

where in the last equality we have made explicit use of the fact that the summand is even with respect to $n$, where $n \in$ integers. Note that this expression comprises an infinite sum over $n$, so that we have also truncated the sum in the last step of Eq. (A1) for numerical purposes. The question that now arises is, how large should $N$ be? And what requirements should it fulfill?

In order to answer these questions, let us plot the results of, say, the reflectance, using several values for $N$. The outcome of such a procedure is shown in Fig. 8. From the figure, one can see a striking difference between the results obtained using odd $N$ values and even $N$ values. In particular, note that whenever we picked $N$ as odd we always got the same (converged) result. This result also coincides with the one in the limit $N \rightarrow \infty$
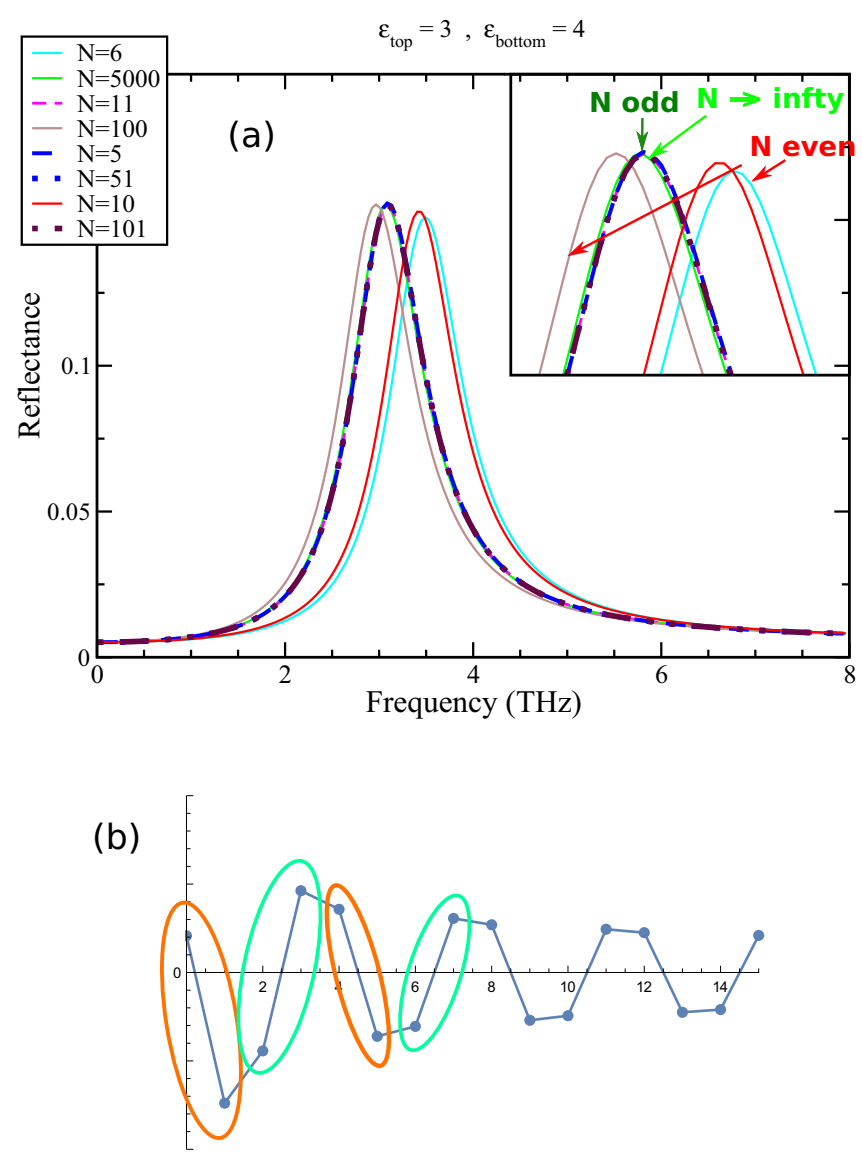

FIG. 8. (a) Reflectance spectra using different values of $N$, which truncates the sum entering in the function $\Lambda(\omega)$. Notice the difference between even and odd $N$ values, in the convergence of results. (b) Alternating series figuring in $\Lambda(\omega)$ [cf. Eq. (A1)] as a function of $n$. 
(light-green curve). Conversely, for even $N$ values, one sees an erratic behavior in the resulting spectra which indicates that the results did not converge. Only for very large values of $N$ even (such has 5000), does one obtain the correct result. Furthermore, notice that even when choosing a small, but odd value for $N$, such as $N=5$, does one get the same (correct) result that one would obtain by choosing a large, but even $N$ instead, such as $N=5000$. This clearly highlights the need to choose $N$ correctly, namely to choose an odd-valued $N$.

The reason for this apparently counterintuitive behavior can be elucidated by plotting the values of the summand in Eq. (A1) as a function of $n$. This is done in Fig. 8(b). Clearly, the figure shows an alternating series; this kind of series usually demands proper care for its accurate computation. In our case, one needs to have special care when choosing $N$; that is, to choose an odd-valued $N$. In that way, the total number of elements of the series is $N+1$ (because it includes the $n=0$ term), which is even, and, therefore, it correctly includes pairs of positive and negative values [as indicated in Fig. 8(b)]. Naturally, as $N$ approaches infinity, the choice between $N$ odd or $N$ even becomes unimportant. However, our analysis demonstrates that, by using an odd-valued $N$, one can obtain accurate results with (odd) $N$ values as small as 5 (this truncation depends naturally on the particular system's geometry, but in general only a few terms of the sum are needed).

\section{Derivation of the formulas for the reflectance and transmittance spectra}

For the derivation of the reflectance and transmittance scattering probabilities, we need to introduce the Poynting vector (for nonmagnetic media),

$$
\mathbf{S}=\frac{1}{\mu_{0}} \mathbf{E} \times \mathbf{B},
$$

which characterizes the flux of electromagnetic energy per unit area. Assuming that both $\mathbf{E}$ and $\mathbf{B}$ can be written as in terms of harmonic functions, $e^{-i \omega t}$, one may write the time-averaged Poynting vector as

$$
\langle\mathbf{S}\rangle=\frac{1}{2 \mu_{0}} \operatorname{Re}\left\{\mathbf{E} \times \mathbf{B}^{*}\right\},
$$

where the star denotes the complex-conjugate. For a TM wave polarized in the $x z$ plane, the time-averaged Poynting vector reads

$$
\langle\mathbf{S}\rangle=\frac{1}{2 \mu_{0}} \operatorname{Re}\left\{E_{x} B_{y}^{*} \hat{\mathbf{z}}-E_{z} B_{y}^{*} \hat{\mathbf{x}}\right\} .
$$

Then, applying the previous equation for the incident wave, one obtains

$$
\left\langle S_{z}^{i}\right\rangle=\frac{c^{2}}{2 \mu_{0} \omega} \operatorname{Re}\left\{\frac{k_{z}}{\epsilon_{1}}\right\}\left|B_{0}^{i}\right|^{2},
$$

for the $z$ component (i.e., the component normal to the graphene grating). Similarly, for the reflected wave one has

$$
\left\langle S_{z, n}^{r}\right\rangle=-\frac{c^{2}}{2 \mu_{0} \omega} \operatorname{Re}\left\{\frac{\kappa_{z, n}^{-}}{\epsilon_{1}}\right\}\left|r_{n}\right|^{2},
$$

for the $n$th diffraction order (or Bloch mode), whereas for the transmitted wave we obtain

$$
\left\langle S_{z, n}^{t}\right\rangle=\frac{c^{2}}{2 \mu_{0} \omega} \operatorname{Re}\left\{\frac{\kappa_{z, n}^{+}}{\epsilon_{2}}\right\}\left|t_{n}\right|^{2} .
$$

At this point we should stress that for purely imaginary wave vectors, $\kappa_{z, n}^{+/-} \rightarrow i\left|\kappa_{z, n}^{+/-}\right|$, the Poynting vectors associated with those Bloch modes give zero contribution [since $\operatorname{Re}\left\{i\left|\kappa_{z, n}^{+/-}\right|\right\}=0$; cf. Eqs. (A6) and (A7)], and, as such, they will not contribute to either the reflectance or the transmittance. This is because they are evanescent waves, and therefore they do not carry energy along the $z$ direction. Notice that the modes are so-called nonpropagating or evanescent whenever $q_{n}>$ $\epsilon k_{0}$, where $q_{n}=k_{x}+n G$ with $G=2 \pi / L$, and $k_{0}=\omega / c$, in which case we have

$$
\kappa_{z, n}^{+/-}=\sqrt{\epsilon_{2 / 1} k_{0}^{2}-q_{n}^{2}} \rightarrow i \sqrt{q_{n}^{2}-\epsilon_{2 / 1} k_{0}^{2}},
$$

in accordance with the definitions used in the main text. Finally, the reflectance and transmittance through the structure under oblique incidence read

$$
R(\omega, \theta)=\sum_{n}\left|\frac{\left\langle S_{z, n}^{r}\right\rangle}{\left\langle S_{z}^{i}\right\rangle}\right|=\frac{\operatorname{Re}\left\{\kappa_{z, 0}^{-} / \epsilon_{1}\right\}}{\operatorname{Re}\left\{k_{z} / \epsilon_{1}\right\}}\left|\frac{r_{0}}{B_{0}^{i}}\right|^{2}
$$

and

$$
T(\omega, \theta)=\sum_{n}\left|\frac{\left\langle S_{z, n}^{t}\right\rangle}{\left\langle S_{z}^{i}\right\rangle}\right|=\frac{\operatorname{Re}\left\{\kappa_{z, 0}^{+} / \epsilon_{2}\right\}}{\operatorname{Re}\left\{k_{z} / \epsilon_{1}\right\}}\left|\frac{t_{0}}{B_{0}^{i}}\right|^{2},
$$

respectively, where the sums were carried out over propagating modes only (solely the $n=0$ mode for the parameters used in this work). The absorption spectrum stems from these equations according to $A(\omega, \theta)=1-R(\omega, \theta)-T(\omega, \theta)$.

For normal incidence, the above formulas simplify considerably to

$$
\begin{gathered}
R(\omega)=\left|\frac{r_{0}}{B_{0}^{i}}\right|^{2}, \\
T(\omega)=\frac{\operatorname{Re}\left\{1 / \sqrt{\epsilon_{2}}\right\}}{\operatorname{Re}\left\{1 / \sqrt{\epsilon_{1}}\right\}}\left|\frac{t_{0}}{B_{0}^{i}}\right|^{2}, \\
A(\omega)=1-\left|\frac{r_{0}}{B_{0}^{i}}\right|^{2}-\frac{\operatorname{Re}\left\{1 / \sqrt{\epsilon_{2}}\right\}}{\operatorname{Re}\left\{1 / \sqrt{\epsilon_{1}}\right\}}\left|\frac{t_{0}}{B_{0}^{i}}\right|^{2},
\end{gathered}
$$

where $r_{0}$ and $t_{0}$ are computed using Eqs. (9) and (15)-(17). Explicitly,

$$
\begin{gathered}
\frac{t_{0}}{B_{0}^{i}}=\frac{\sqrt{\epsilon_{2}}}{\sqrt{\epsilon_{1}}+\sqrt{\epsilon_{2}}}\left[2-\mu_{0} \frac{\chi}{B_{0}^{i}} \frac{\pi w^{2}}{8 L}\right], \\
\frac{r_{0}}{B_{0}^{i}}=1-\frac{2 \sqrt{\epsilon_{1}}}{\sqrt{\epsilon_{1}}+\sqrt{\epsilon_{2}}}+\mu_{0} \frac{\chi}{B_{0}^{i}} \frac{\pi w^{2}}{8 L} \frac{\sqrt{\epsilon_{1}}}{\sqrt{\epsilon_{1}}+\sqrt{\epsilon_{2}}},
\end{gathered}
$$

with

$$
\begin{gathered}
\frac{\chi}{B_{0}^{i}}=\frac{2 \kappa_{z, 0}^{+} \kappa_{z, 0}^{-}}{\epsilon_{1} \kappa_{z, 0}^{+}+\epsilon_{2} \kappa_{z, 0}^{-}} \frac{\sigma(\omega) c^{2}}{\omega} \frac{1}{\Lambda(\omega)}, \\
\Lambda(\omega)=\frac{w}{4} \sum_{n=-\infty}^{\infty} \frac{1}{n} J_{1}(n \pi w / L)\left[1+\frac{\sigma(\omega)}{\omega \epsilon_{0}} \frac{\kappa_{z, n}^{+} \kappa_{z, n}^{-}}{\epsilon_{1} \kappa_{z, n}^{+}+\epsilon_{2} \kappa_{z, n}^{-}}\right] .
\end{gathered}
$$


(a)
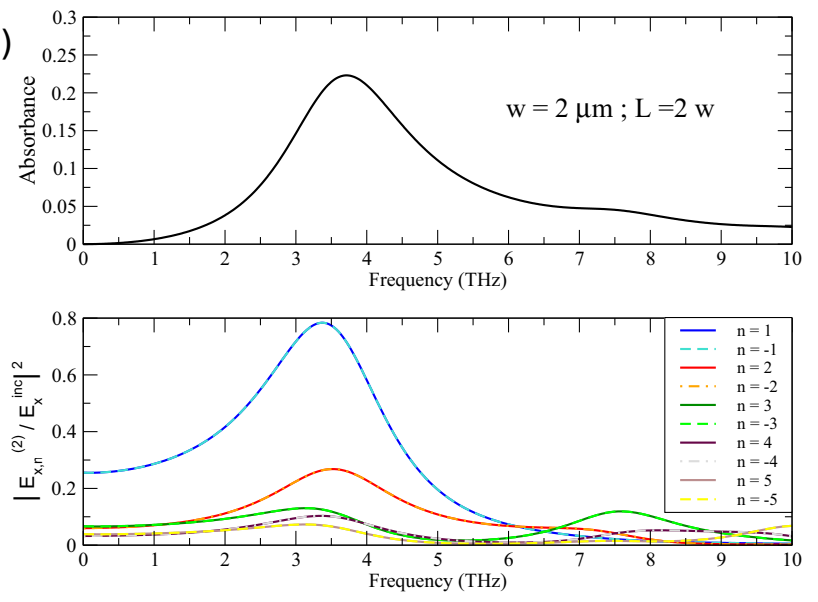

(b)
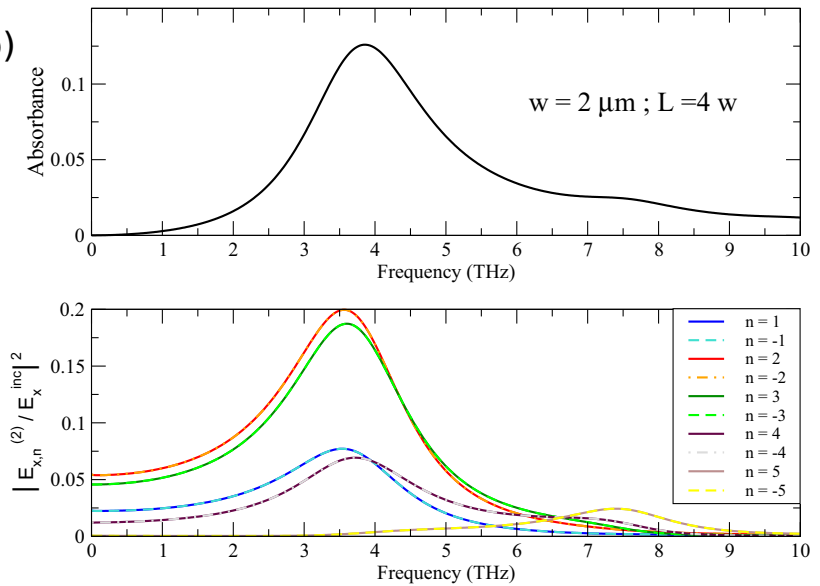

FIG. 9. Absorbance and relative modulus-squared Bloch amplitudes $\left|E_{x, n}^{(2)}\right|^{2}$ corresponding to several Bragg modes, for two different configurations: one with a filling ratio of $w / L=1 / 2$ (top) and another with $w / L=1 / 4$ (bottom).

\section{Modes contributing to the fundamental resonance for different filling ratios}

We further note that the particular Bragg modes that couple the most to the fundamental GSP resonance depend on the specific filling ratio of the system. Such dependence is a consequence of the overlapping of the fields with the ribbon, and, therefore, the geometric configuration (the filling ratio) is an important parameter. As an example, in Fig. 9 (obtained using the semi-analytical method outlined in appendix C) we show relative modulus-squared Bloch amplitudes $\left|E_{x, n}^{(2)}\right|^{2}$ corresponding to several Bragg modes, for two different configurations.

\section{Spectral weight akin to the higher-order multipolar resonances}

Figure 10 shows the two lowest-energy plasmonic resonances in the system under study (for different doping levels). Note that, as discussed in the main article, the analytic approach does not account for the higher-order (multipolar) resonances. The semianalytical method, on the other hand, includes these resonances; notice, for instance, the small,

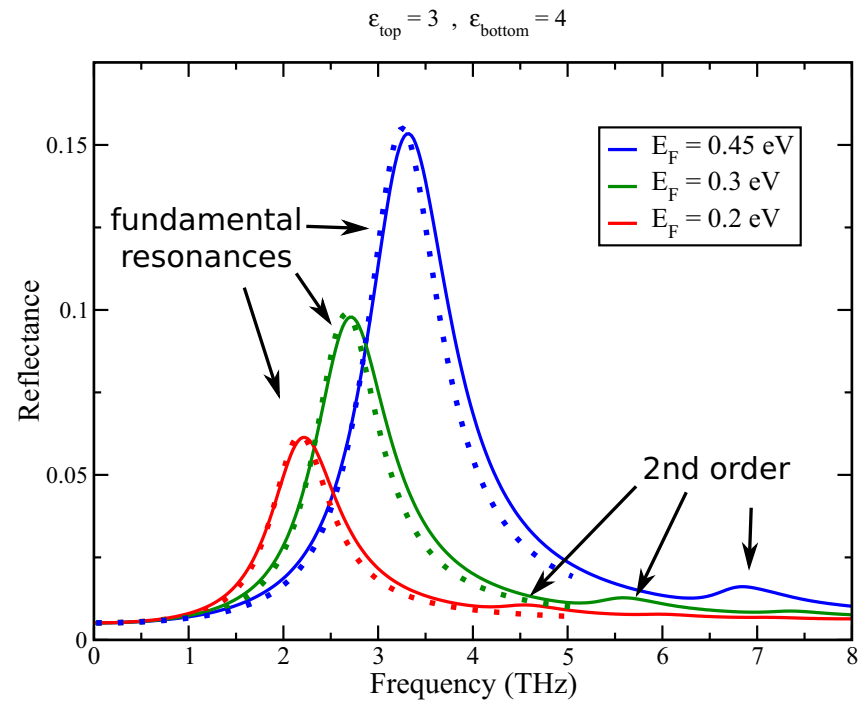

FIG. 10. GSP resonances in a periodic grid of graphene ribbons ( $w=4 \mu \mathrm{m}$ and $L=2 w$ ) at different carrier concentrations. The solid lines correspond to the results obtained using the semianalytical method whereas the dotted lines correspond to the outcome of the full-analytical approach.

weaker peaks at higher frequencies to the right of the fundamental resonance. These, however, carry little spectral weight and can only be seen due to the rather small damping parameter $(\Gamma=2.6 \mathrm{meV})$. This justifies their omission when using the full-analytical technique, as their contribution is rather small.

\section{Ansatz for the current}

In Fig. 11, we compare the ansatz for the current, cf. Eq. (11), against the same quantity computed as a Fourier series, as in the semianalytical method [see Eq. (C7)]. Notice that the overall correspondence is quite good, thus providing further evidence for the validity of the edge condition in our fully analytic framework.

The oscillations emerging in the plot corresponding to the semi-analytic method are a natural consequence of the use of

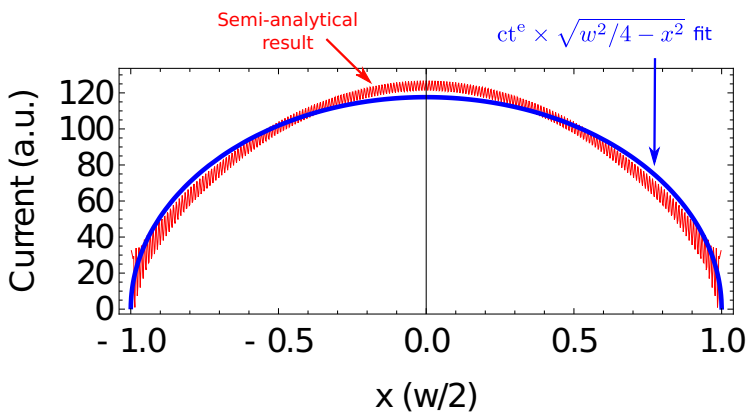

FIG. 11. Current, $J_{x}(x)$, within the graphene stripes obtained using the semianalytical method (Fourier expansion) and corresponding fitting function of the type $c t^{e} \times \sqrt{w^{2} / 4-x^{2}}$ to illustrate the approximation which is made when employing the edge condition [ansatz for the current; see Eq. (11)]. 
a Fourier expansion to describe the current in such geometry, a feature that is known as Gibbs phenomenon [92]. Naturally, these are absent in the analytic ansatz for the current.

\section{APPENDIX B: DYNAMICAL CONDUCTIVITY OF GRAPHENE}

In this work we model the dynamical conductivity of the graphene ribbons using Kubo's formula within the local approximation at room temperature $(T=300 \mathrm{~K})$. In such framework, the material's $2 \mathrm{D}$ conductivity reads [24,93]

$$
\begin{aligned}
& \sigma_{\text {Kubo }}(\omega)=\sigma_{\text {intra }}(\omega)+\sigma_{\text {inter }}(\omega) \text {, } \\
& \sigma_{\text {intra }}(\omega)=\frac{\sigma_{0}}{\pi} \frac{4}{\Gamma-i \hbar \omega}\left[E_{F}+\frac{2}{\beta} \ln \left(1+e^{-\beta E_{F}}\right)\right] \text {, } \\
& \sigma_{\text {inter }}(\omega)=\frac{\sigma_{0}}{\pi}[\pi G(\hbar \omega / 2) \\
& \left.+i 4 \hbar \omega \int_{0}^{\infty} d E \frac{G(E)-G(\hbar \omega / 2)}{(\hbar \omega)^{2}-4 E^{2}}\right],
\end{aligned}
$$

where $\beta=\left(k_{B} T\right)^{-1}$ (here $k_{B}$ is Boltzmann's constant), $\sigma_{0}=$ $e^{2} /(4 \hbar)$, and where the quantity $G(x)$ is defined as

$$
G(x)=\frac{\sinh (x \beta)}{\cosh \left(E_{F} \beta\right)+\cosh (x \beta)} .
$$

In the $\mathrm{THz}$ regime, for graphene under typical doping levels-such that $E_{F} \gg k_{B} T$ and $2 E_{F}>\hbar \omega$-the conductivity of graphene can be well approximated by the Drude-like expression

$$
\sigma_{\mathrm{D}}(\omega) \approx \frac{\sigma_{0}}{\pi} \frac{4 E_{F}}{\Gamma-i \hbar \omega},
$$

provided that the conditions $E_{F} \gg k_{B} T$ and $2 E_{F}>\hbar \omega$ are met.

\section{APPENDIX C: SEMIANALYTICAL METHOD IN A NUTSHELL}

Similarly to the fully analytical method described in the main text, the semianalytical method also expresses the EM fields in the form of Bloch sums [22,24,75-77]. Namely, the fields in the medium $j$ may be written as (under normal incidence)

$$
\begin{aligned}
& \mathcal{E}_{x}^{(j)}(x, z)=E_{x}^{i n c} e^{i k_{z} z} \delta_{j, 1}+\sum_{n} E_{x, n}^{(j)} e^{i n G x-\xi_{j, n}|z|}, \\
& \mathcal{E}_{z}^{(j)}(x, z)=E_{z}^{i n c} e^{i k_{z} z} \delta_{j, 1}+\sum_{n}^{n} E_{z, n}^{(j)} e^{i n G x-\xi_{j, n}|z|}, \\
& \mathcal{B}_{y}^{(j)}(x, z)=B_{y}^{i n c} e^{i k_{z} z} \delta_{j, 1}+\sum_{n} B_{y, n}^{(j)} e^{i n G x-\xi_{j, n}|z|},
\end{aligned}
$$

where $G=2 \pi / L, k_{z}=\sqrt{\epsilon_{1}} k_{0}$, and $\xi_{j, n}^{2}=(n G)^{2}-\epsilon_{j} k_{0}^{2}$. Imposing the adequate boundary conditions, one obtains the following system of equations

$$
\begin{gathered}
Q_{0} E_{x, 0}^{(2)}+\frac{i}{\omega \epsilon_{0}} \sum_{l} \tilde{\sigma}_{-l} E_{x, l}^{(2)}=i \frac{2 \epsilon_{1}}{k_{z}} E_{x}^{i n c}, \\
Q_{n} E_{x, n}^{(2)}+\frac{i}{\omega \epsilon_{0}} \sum_{l} \tilde{\sigma}_{n-l} E_{x, l}^{(2)}=0 .
\end{gathered}
$$

for $n=0$ and $n \neq 0$, respectively, and where $Q_{n}=\epsilon_{1} / \xi_{1, n}+$ $\epsilon_{2} / \xi_{2, n}$. In Eqs. (C4) and (C5), the quantities $\tilde{\sigma}_{m}$ are the components of the Fourier series that incorporates the system's periodicity, that is

$$
\begin{gathered}
\sigma(x)=\sum_{m} \tilde{\sigma}_{m} e^{i m G x}, \\
\tilde{\sigma}_{m}=\frac{1}{L} \int_{-L / 2}^{L / 2} \sigma(x) e^{-i m G x} d x .
\end{gathered}
$$

The numerical solution of the (truncated) system of equations posed by Eqs. (C4) and (C5) for each frequency $\omega$ (entering as a parameter), renders the field amplitudes, $E_{x, l}^{(2)}$, in terms of $E_{x}^{\text {inc }}$. As before, only the mode with $n=0$ is propagating, and thus only this contributes (i.e., reaches the far-field) to the transmittance, reflectance, and absorbance, which read

$$
\begin{gathered}
\mathcal{T}(\omega)=\frac{\operatorname{Re}\left\{\sqrt{\epsilon_{2}}\right\}}{\operatorname{Re}\left\{\sqrt{\epsilon_{1}}\right\}}\left|\frac{E_{x, 0}^{(2)}}{E_{x}^{i n c}}\right|^{2}, \\
\mathcal{R}(\omega)=\left|\frac{E_{x, 0}^{(2)}-E_{x}^{i n c}}{E_{x}^{i n c}}\right|^{2}, \\
\mathcal{A}(\omega)=1-\mathcal{T}(\omega)-\mathcal{R}(\omega) .
\end{gathered}
$$

From these expressions, the corresponding spectra akin to the semianalytical model may be readily obtained.
[1] A. A. Maradudin, W. L. Barnes, and J. R. Sambles, Modern Plasmonics (Elsevier, Amsterdam, 2014).

[2] W. L. Barnes, A. Dereux, and T. W. Ebbesen, Nature (London) 424, 824 (2003).

[3] D. K. Gramotnev and S. I. Bozhevolnyi, Nat. Photonics 4, 83 (2010).

[4] J. A. Schuller, E. S. Barnard, W. Cai, Y. C. Jun, J. S. White, and M. L. Brongersma, Nat. Mater. 9, 193 (2010).

[5] S. Armstrong, Nat. Photonics 6, 720 (2012).

[6] E. Ozbay, Science 311, 189 (2006).
[7] A. A. Toropov and T. V. Shubina, Plasmonic Effects in Metal-Semiconductor Nanostructures (Oxford University Press, Oxford, 2015).

[8] M. Pelton, J. Aizpurua, and G. Bryant, Laser Photon. Rev. 2, 136 (2008).

[9] M. Pelton and G. W. Bryant, Introduction to Metal-Nanoparticle Plasmonics, 1st ed. (John Wiley \& Sons, Hoboken, NJ, 2013).

[10] L. Billings, Nature (London) 500, 138 (2013).

[11] N. I. Zheludev and Y. S. Kivshar, Nat. Mater. 11, 917 (2012).

[12] A. K. Geim, Science 324, 1530 (2009). 
[13] A. H. C. Neto, F. Guinea, N. M. R. Peres, K. S. Novoselov, and A. K. Geim, Rev. Mod. Phys. 81, 109 (2009).

[14] A. C. Ferrari et al., Nanoscale 7, 4598 (2015).

[15] P. Avouris, Nano Lett. 10, 4285 (2010).

[16] F. Bonaccorso, Z. Sun, T. Hasan, and A. C. Ferrari, Nat. Photonics 4, 611 (2010).

[17] A. N. Grigorenko, M. Polini, and K. S. Novoselov, Nat. Photonics 6, 749 (2012).

[18] F. J. G. de Abajo, ACS Photonics 1, 135 (2014).

[19] S. Xiao, X. Zhu, B.-H. Li, and N. A. Mortensen, Front. Phys. 11, 117801 (2016).

[20] T. Low and P. Avouris, ACS Nano 8, 1086 (2014).

[21] F. H. L. Koppens, D. E. Chang, and F. J. G. de Abajo, Nano Lett. 11, 3370 (2011).

[22] Y. V. Bludov, A. Ferreira, N. M. R. Peres, and M. I. Vasilevskiy, Int. J. Mod. Phys. B 27, 1341001 (2013).

[23] A. Politano and G. Chiarello, Nanoscale 6, 10927 (2014).

[24] P. A. D. Gonçalves and N. M. R. Peres, An Introduction to Graphene Plasmonics (World Scientific, Singapore, 2016).

[25] J. B. Khurgin and A. Boltasseva, MRS Bull. 37, 768 (2012).

[26] D. Rodrigo, O. Limaj, D. Janner, D. Etezadi, F. J. G. de Abajo, V. Pruneri, and H. Altug, Science 349, 165 (2015).

[27] D. B. Farmer, P. Avouris, Y. Li, T. F. Heinz, and S.-J. Han, ACS Photonics 3, 553 (2016).

[28] P. Wang, O. Liang, W. Zhang, T. Schroeder, and Y.-H. Xie, Adv. Mater. 25, 4918 (2013).

[29] S. Zeng, S. Hu, J. Xia, T. Anderson, X.-Q. Dinh, X.-M. Meng, P. Coquet, and K.-T. Yong, Sens. Actuators B: Chem. 207, 801 (2015).

[30] Y. Zhao, X. Hu, G. Chen, X. Zhang, Z. Tan, J. Chen, R. S. Ruoff, Y. Zhu, and Y. Lu, Phys. Chem. Chem. Phys. 15, 17118 (2013).

[31] X. Ling, L. Xie, Y. Fang, H. Xu, H. Zhang, J. Kong, M. S. Dresselhaus, J. Zhang, and Z. Liu, Nano Lett. 10, 553 (2010).

[32] W. Xu, X. Ling, J. Xiao, M. S. Dresselhaus, J. Kong, H. Xu, Z. Liu, and J. Zhang, Proc. Natl. Acad. Sci. USA 109, 9281 (2012).

[33] Y. Zhao, G. Chen, Y. Du, J. Xu, S. Wu, Y. Qu, and Y. Zhu, Nanoscale 6, 13754 (2014).

[34] C.-Y. Liu, K.-C. Liang, W. Chen, C. hao Tu, C.-P. Liu, and Y. Tzeng, Opt. Express 19, 17092 (2011).

[35] S. Heeg, R. Fernandez-Garcia, A. Oikonomou, F. Schedin, R. Narula, S. A. Maier, A. Vijayaraghavan, and S. Reich, Nano Lett. 13, 301 (2013).

[36] A. Urich, A. Pospischil, M. M. Furchi, D. Dietze, K. Unterrainer, and T. Mueller, Appl. Phys. Lett. 101, 153113 (2012).

[37] Q. Bao, H. Zhang, B. Wang, Z. Ni, C. H. Y. X. Lim, Y. Wang, D. Y. Tang, and K. P. Loh, Nat. Photonics 5, 411 (2011).

[38] C. Sorger, S. Preu, J. Schmidt, S. Winnerl, Y. V. Bludov, N. M. R. Peres, M. I. Vasilevskiy, and H. B. Weber, New J. Phys. 17, 053045 (2015).

[39] Z. Sun, A. Martinez, and F. Wang, Nat. Photonics 10, 227 (2016).

[40] B. Sensale-Rodriguez, R. Yan, M. Zhu, D. Jena, L. Liu, and H. Grace Xing, Appl. Phys. Lett. 101, 261115 (2012).

[41] R. Yu, V. Pruneri, and F. J. G. de Abajo, ACS Photonics 2, 550 (2015).

[42] B. Sensale-Rodriguez, S. Rafique, R. Yan, M. Zhu, V. Protasenko, D. Jena, L. Liu, and H. G. Xing, Opt. Express 21, 2324 (2013).

[43] Z. Fang, Z. Liu, Y. Wang, P. M. Ajayan, P. Nordlander, and N. J. Halas, Nano Lett. 12, 3808 (2012).
[44] Y. Liu, R. Cheng, L. Liao, H. Zhou, J. Bai, G. Liu, L. Liu, Y. Huang, and X. Duan, Nat. Commun. 2, 579 (2011).

[45] Z. Sun, L. Aigouy, and Z. Chen, Nanoscale 8, 7377 (2016).

[46] T. J. Echtermeyer, S. Milana, U. Sassi, A. Eiden, M. Wu, E. Lidorikis, and A. Ferrari, Nano Lett. 16, 8 (2016).

[47] F. H. L. Koppens, T. Mueller, P. Avouris, A. C. Ferrari, M. S Vitiello, and M. Polini, Nat. Nanotechnol. 9, 780 (2014).

[48] L. Ju, B. Geng, J. Horng, C. Girit, M. Martin, Z. Hao, H. A. Bechtel, X. Liang, A. Zettl, Y. R. Shen, and F. Wang, Nat. Nanotechnol. 6, 630 (2011).

[49] H. Yan, T. Low, W. Zhu, Y. Wu, M. Freitag, X. Li, F. Guinea, P. Avouris, and F. Xia, Nat. Photonics 7, 394 (2013).

[50] P. Nene, J. H. Strait, W.-M. Chan, C. Manolatou, J. W. Kevek, S. Tiwari, P. L. McEuen, and F. Rana, Appl. Phys. Lett. 105, 143108 (2014).

[51] H. Yan, X. Li, B. Chandra, G. Tulevski, Y. Wu, M. Freitag, W. Zhu, P. Avouris, and F. Xia, Nat. Nanotechnol. 7, 330 (2012).

[52] I. J. Luxmoore, C. H. Gan, P. Q. Liu, F. Valmorra, P. Li, J. Faist, and G. R. Nash, ACS Photonics 1, 1151 (2014).

[53] H. Hu, F. Zhai, D. Hu, Z. Li, B. Bai, X. Yang, and Q. Dai, Nanoscale 7, 19493 (2015).

[54] H. Yan, F. Xia, Z. Li, and P. Avouris, New J. Phys. 14, 125001 (2012).

[55] Z. Fang, S. Thongrattanasiri, A. Schlather, Z. Liu, L. Ma, Y. Wang, P. M. Ajayan, P. Nordlander, N. J. Halas, and F. J. G. de Abajo, ACS Nano 7, 2388 (2013).

[56] Z. Fang, Y. Wang, A. E. Schlather, Z. Liu, P. M. Ajayan, F. J. G. de Abajo, P. Nordlander, X. Zhu, and N. J. Halas, Nano Lett. 14, 299 (2014).

[57] X. Zhu, W. Wang, W. Yan, M. B. Larsen, P. Boeggild, T. G. Pedersen, S. Xiao, J. Zi, and N. A. Mortensen, Nano Lett. 14, 2907 (2014).

[58] P. Q. Liu, F. Valmorra, C. Maissen, and J. Faist, Optica 2, 135 (2015).

[59] K. Y. M. Yeung, J. Chee, H. Yoon, Y. Song, J. Kong, and D. Ham, Nano Lett. 14, 2479 (2014).

[60] V. W. Brar, M. S. Jang, M. Sherrott, J. J. Lopez, and H. A. Atwater, Nano Lett. 13, 2541 (2013).

[61] V. W. Brar, M. S. Jang, M. Sherrott, S. Kim, J. J. Lopez, L. B. Kim, M. Choi, and H. Atwater, Nano Lett. 14, 3876 (2014).

[62] Y. Yao, M. A. Kats, P. Genevet, N. Yu, Y. Song, J. Kong, and F. Capasso, Nano Lett. 13, 1257 (2013).

[63] Z. Fang, Y. Wang, Z. Liu, A. Schlather, P. M. Ajayan, F. H. L. Koppens, P. Nordlander, and N. J. Halas, ACS Nano 6, 10222 (2012).

[64] P. Alonso-González et al., Science 344, 1369 (2014).

[65] W. Gao, G. Shi, Z. Jin, J. Shu, Q. Zhang, R. Vajtai, P. M. Ajayan, J. Kono, and Q. Xu, Nano Lett. 13, 3698 (2013).

[66] X. Zhu, W. Yan, P. Uhd Jepsen, O. Hansen, N. Asger Mortensen, and S. Xiao, Appl. Phys. Lett. 102, 131101 (2013).

[67] Z. Fei et al., Nano Lett. 11, 4701 (2011).

[68] H. Yan, Z. Li, X. Li, W. Zhu, P. Avouris, and F. Xia, Nano Lett. 12, 3766 (2012).

[69] T. J. Constant, S. M. Hornett, D. E. Chang, and E. Hendry, Nat. Phys. 12, 124 (2016).

[70] M. Tokman, Y. Wang, I. Oladyshkin, A. R. Kutayiah, and A. Belyanin, Phys. Rev. B 93, 235422 (2016).

[71] Z. Fei et al., Nature (London) 487, 82 (2012).

[72] J. Chen et al., Nature (London) 487, 77 (2012).

[73] A. Woessner et al., Nat. Mater. 14, 421 (2015). 
[74] I. D. Barcelos, A. R. Cadore, L. C. Campos, A. Malachias, K. Watanabe, T. Taniguchi, F. C. B. Maia, R. Freitas, and C. Deneke, Nanoscale 7, 11620 (2015).

[75] N. M. R. Peres, A. Ferreira, Y. V. Bludov, and M. I. Vasilevskiy, J. Phys.: Condens. Matter 24, 245303 (2012).

[76] Y. V. Bludov, N. M. R. Peres, and M. I. Vasilevskiy, Phys. Rev. B 85, 245409 (2012).

[77] T. M. Slipchenko, M. L. Nesterov, L. Martin-Moreno, and A. Y. Nikitin, J. Opt. 15, 114008 (2013).

[78] S. Thongrattanasiri, A. Manjavacas, and F. J. G. de Abajo, ACS Nano 6, 1766 (2012).

[79] T. Christensen, W. Wang, A.-P. Jauho, M. Wubs, and N. A. Mortensen, Phys. Rev. B 90, 241414 (2014).

[80] M. Abramowitz and I. A. Stegun, Handbook of Mathematical Functions: With Formulas, Graphs, and Mathematical Tables (Dover, New York, 1965).

[81] K. Barkeshli, Advanced Electromagnetics and Scattering Theory (Springer, New York, 2015).

[82] S. A. Mikhailov and N. A. Savostianova, Phys. Rev. B 71, 035320 (2005).
[83] V. N. Kotov, B. Uchoa, V. M. Pereira, F. Guinea, and A. H. Castro Neto, Rev. Mod. Phys. 84, 1067 (2012).

[84] Z. Q. Li, E. A. Henriksen, Z. Jiang, Z. Hao, M. C. Martin, P. Kim, H. L. Stormer, and D. N. Basov, Nat. Phys. 4, 532 (2008).

[85] J. Christensen, A. Manjavacas, S. Thongrattanasiri, F. H. L. Koppens, and F. J. G. de Abajo, ACS Nano 6, 431 (2012).

[86] T. R. A. Bondeson and P. Ingelström, Computational Electromagnetics (Springer, New York, 2005).

[87] R. Fuchs and K. L. Kliewer, Phys. Rev. 140, A2076 (1965).

[88] G. D. Mahan, Many-Particle Physics, 3rd ed. (Springer, New York, 2000).

[89] N. W. Ashcroft and N. D. Mermin, Solid State Physics, 1st ed. (Saunders College, New York, 1976).

[90] R. Kitamura, L. Pilon, and M. Jonasz, Appl. Opt. 46, 8118 (2007).

[91] M. Y. Han, B. Özyilmaz, Y. Zhang, and P. Kim, Phys. Rev. Lett. 98, 206805 (2007).

[92] H. J. W. George B. Arfken and F. E. Harris, Mathematical Methods for Physicists, 7th ed. (Elsevier, New York, 2013).

[93] L. A. Falkovsky, J. Phys.: Conf. Ser. 129, 012004 (2008). 